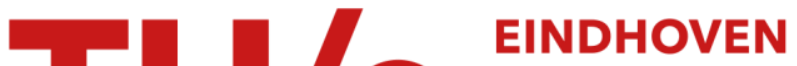 UNIVERSITY OF TECHNOLOGY
}

\section{Three-Dimensional Modeling of Organic Light-Emitting Diodes Containing Molecules with Large Electric Dipole Moments}

Citation for published version (APA):

Coehoorn, R., Lin, X., Weijtens, C. H. L., Gottardi, S., \& van Eersel, H. (2021). Three-Dimensional Modeling of Organic Light-Emitting Diodes Containing Molecules with Large Electric Dipole Moments. Physical Review Applied, 16(3), [034048]. https://doi.org/10.1103/PhysRevApplied.16.034048

DOI:

10.1103/PhysRevApplied.16.034048

Document status and date:

Published: 01/09/2021

\section{Document Version:}

Publisher's PDF, also known as Version of Record (includes final page, issue and volume numbers)

\section{Please check the document version of this publication:}

- A submitted manuscript is the version of the article upon submission and before peer-review. There can be important differences between the submitted version and the official published version of record. People interested in the research are advised to contact the author for the final version of the publication, or visit the $\mathrm{DOI}$ to the publisher's website.

- The final author version and the galley proof are versions of the publication after peer review.

- The final published version features the final layout of the paper including the volume, issue and page numbers.

Link to publication

\section{General rights}

Copyright and moral rights for the publications made accessible in the public portal are retained by the authors and/or other copyright owners and it is a condition of accessing publications that users recognise and abide by the legal requirements associated with these rights.

- Users may download and print one copy of any publication from the public portal for the purpose of private study or research.

- You may not further distribute the material or use it for any profit-making activity or commercial gain

- You may freely distribute the URL identifying the publication in the public portal.

If the publication is distributed under the terms of Article 25fa of the Dutch Copyright Act, indicated by the "Taverne" license above, please follow below link for the End User Agreement:

www.tue.nl/taverne

Take down policy

If you believe that this document breaches copyright please contact us at:

openaccess@tue.nl

providing details and we will investigate your claim. 


\title{
Three-Dimensional Modeling of Organic Light-Emitting Diodes Containing Molecules with Large Electric Dipole Moments
}

\author{
R. Coehoorn $\odot,{ }^{1,2,3, *}$ X. Lin, ${ }^{3}$ C.H.L. Weijtens $\odot,{ }^{1}$ S. Gottardi, ${ }^{4}$ and H. van Eersel ${ }^{4}$ \\ ${ }^{1}$ Department of Applied Physics, Eindhoven University of Technology, P.O. Box 513, MB Eindhoven NL-5600, \\ Netherlands \\ ${ }^{2}$ Institute for Complex Molecular Systems, Eindhoven University of Technology, P.O. Box 513, MB Eindhoven \\ NL-5600, Netherlands \\ ${ }^{3}$ Guangdong Provincial Key Laboratory of Optical Information Materials and Technology and Institute of \\ Electronic Paper Displays, South China Academy of Advanced Optoelectronics, South China Normal University, \\ Guangzhou 510006, People's Republic of China \\ ${ }^{4}$ Simbeyond B.V., Het Eeuwsel 57, AS Eindhoven 5612, Netherlands
}

(Received 29 June 2021; revised 24 August 2021; accepted 2 September 2021; published 28 September 2021)

\begin{abstract}
We present the results of a three-dimensional kinetic Monte Carlo (3D KMC) simulation study of the current density and efficiency of a prototypical green phosphorescent organic light-emitting diode within which the TPBi $\left[2,2^{\prime}, 2^{\prime \prime}-(1,3,5\right.$-benzinetriyl)-tris(1-phenyl-1- $H$-benzimidazole)] molecules that comprise the electron transport layer have a large $(7 \mathrm{D})$ static dipole moment. By explicitly calculating the dipole-induced electrostatic field distribution the additional energetic disorder and the additional internal electrostatic field due to the small net dipole-moment orientation in TPBi are included in a mechanistic manner. The simulation results are compared with the results of an experimental study by B. Sim et al. [ACS Appl. Mater. Interf. 8, 33010 (2016)]. Using a set of simulation parameters that provides a fair agreement with the experimental voltage-dependent current density, external quantum efficiency, and emission profiles, the simulations are used to study the sensitivity to the size and net orientation of the dipole moments. We show how the dipole-induced disorder and the dipole orientation affect the current density, the electron injection efficiency, the blocking of holes by the TPBi layer, the shape of the emission profile, and the quantum efficiency. A key element of the validation of the simulations is a comparison with measured emission profiles, which Sim et al. obtained from sense layer experiments. Explicit KMC simulations of these experiments are used to investigate the expected accuracy with which the emission profiles are probed.
\end{abstract}

DOI: 10.1103/PhysRevApplied.16.034048

\section{INTRODUCTION}

The use of molecules with large static dipole moments can strongly affect the functioning of organic light-emitting diodes (OLEDs). Firstly, in disordered materials the stray electric fields from randomly oriented dipole moments gives rise to a large contribution to the width of the frontier orbital density of states (DOS), and hence to a strong reduction of the mobility [1-4]. A specific consequence of dipolar-induced disorder is an enhanced electric field dependence of the mobility, resulting from spatial correlation of the energy levels due to the long-range nature of the dipolar fields [5-8]. Secondly, already a small degree of orientational ordering, obtained upon thermal evaporation deposition, can lead to a large net dipole moment of a layer ["giant surface potential" (GSP) or "spontaneous orientation polarization" (SOP) effect]. This modifies the electric

\footnotetext{
*r.coehoorn@tue.nl
}

field across the device thickness, affects charge injection from an electrode and transport across internal interfaces [9-15], can lead to an enhanced exciton dissociation rate [16], and can already below the turn-on voltage lead to enhanced exciton quenching due the interaction with accumulated charges at the interface between an emissive layer and a charge transport layer with a large GSP effect [17]. Finally, phosphorescent emitter molecules with large static dipole moments that are present at small concentrations in a blend show a tendency to aggregate during thin-film deposition, leading to a red-shifted emission and to an enhanced efficiency loss due to triplet-triplet annihilation $[18,19]$.

In the past decade, three-dimensional kinetic Monte Carlo (3D KMC) simulations have been used to model the device performance of OLEDs utilizing phosphorescence [20-24], fluorescence $[25,26]$, thermally activated delayed fluorescence [26], and hyperfluorescence [26]. In most studies, the molecules are assumed to reside on a 
simple cubic lattice ("lattice KMC"), and their electronic (frontier orbital) and excitonic energies are chosen randomly from a material-specific distribution function. So far, in such simulations the effect of static dipole moments has only been included by taking the resulting enhancement of the width of the DOS and the resulting spatial correlation into account, and only for unipolar singlelayer devices [27]. Kordt et al. have carried out off-lattice KMC simulations of the hole transport in unipolar singlelayer devices based on molecules with large static dipole moments, after first calculating the thin-film morphology using force-field methods [23]. However, such an ab initio approach for obtaining the energy levels, their spatial correlation, and the pair-specific interaction energies that determine the local hopping rates, is computationally very demanding and is only feasible for systems with a relatively small number of molecules (up to approximately $10^{4}$ ). It is therefore of interest to investigate how well the effects of dipole moments and their partial orientation on the device performance of OLEDs can already be predicted accurately from relatively efficient lattice KMC simulations.

Here, we present the results of a 3D KMC study of the device performance of archetypical phosphorescent OLEDs in which the electron transport layer (ETL) is formed by the organic semiconductor TPBi $\left[2,2^{\prime}\right.$, $2^{\prime \prime}$-(1,3,5-benzinetriyl)-tris(1-phenyl-1- $H$-benzimidazole)], which has a relatively large dipole moment of $p \cong 7 \mathrm{D}$ [12]. TPBi has been used frequently in OLEDs as an electron-transporting material and as a host material for fluorescent and phosphorescent dopants [20,28-32]. The molecular structure of TPBi is shown in Fig. 1(a). The dipole moment is a result of a dihedral out-of-plane rotation of the three polar ligands, leading to a propellertype molecular shape [12]. Figure 2 shows the layer and energy-level structure of the device that is studied. The emissive layer (EML) consists of $6.5 \mathrm{~mol} \%$ of the yellowgreen emitter $\operatorname{Ir}(\text { ppy })_{2}$ (acac) \{bis[2-(2-pyridinyl- $N$ )phenylC] (acetylacetonato)iridium(III) $\}$, embedded in CBP $\left(4,4^{\prime}\right.$ $N, N^{\prime}$-dicarbazole-1,1'-biphenyl). CBP is also used as the hole transport material in the hole transport layer (HTL). These OLEDs show a high maximum external quantum efficiency (EQE, $\eta_{\mathrm{EQE}}$ ), approximately $23 \%-24 \%$ [33,34]. When combined with a high-refractive index anode layer that enhances the light outcoupling efficiency, flexible OLEDs with the same functional layer structure even show a maximum EQE of approximately 40\% [35]. The use of a partial homojunction device structure, with CBP as HTL and as the host in the EML, not only simplifies the device fabrication process but also the device simulations, viz. by eliminating uncertainties with respect to frontier orbital energy-level offsets at the HTL | EML interface. Notable additional motivating factors for selecting this device are (i) that a comparison of the results from various types of experiments leads to a consistent value
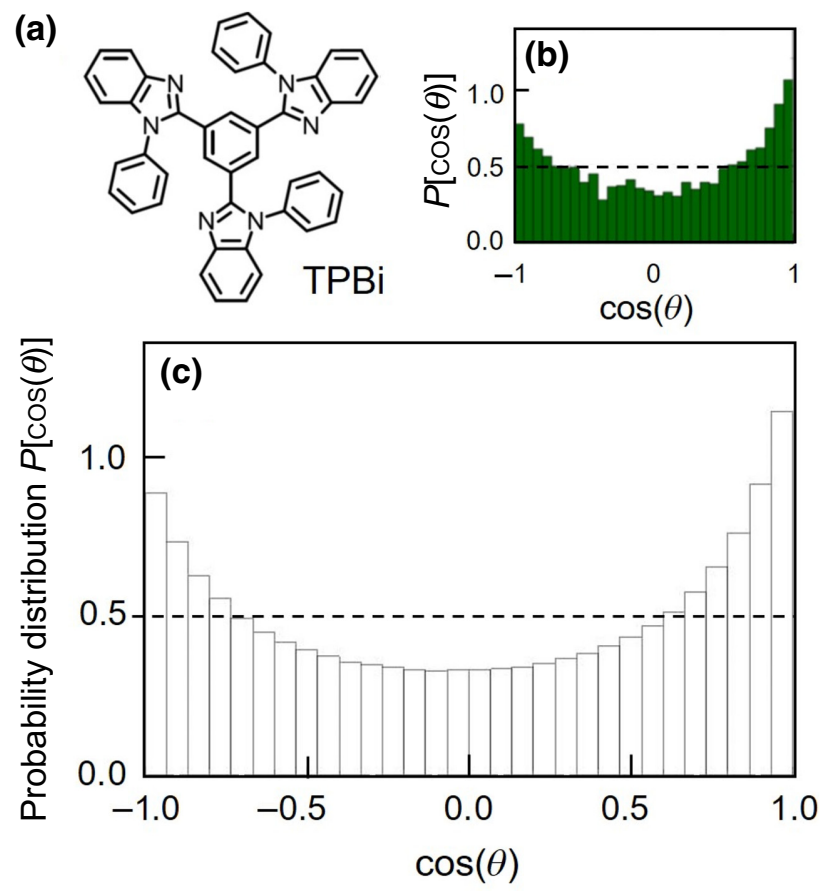

FIG. 1. Molecular structure of TPBi (a) and angular dependence of the normalized orientation probability distribution function for TPBi, obtained from quantum-chemical calculations in Ref. [12] (b) and obtained from a fit to these data using the approach described in Sec. II B (c). A three-dimensional representation of the structure of TPBi has been given in Fig. 2(a) of Ref. [12].

of the degree of dipole orientation in TPBi, viz. approximately $5 \%$ (see Sec. III), (ii) that the other two materials used have no (CBP) or a relatively small $\left[\operatorname{Ir}(\mathrm{ppy})_{2}\right.$ (acac), 1.6 D] static dipole moment [36] that can be neglected in this study, and (iii) that an accurate analysis of the light outcoupling efficiency is available [34], so that the measured $\mathrm{EQE}$ can be related to the internal quantum efficiency (IQE).

In our analysis, we compare the calculated current density versus voltage curve, $J(V)$, the roll-off curve, $\eta_{\mathrm{IQE}}(J)$, and the $J$-dependent emission profiles with the experimental results that have been reported in Ref. [34]. In that study, the current density dependence of the emission profile was obtained from sense-layer experiments, in which very thin layers of a red-emitting fluorescent dye molecule were added at a very small concentration at different positions in the EML. We carry out 3D KMC simulations that precisely mimic these experiments, and show that the emission profile that follows from such experiments is similar but not precisely the same as the actual emission profile.

The paper is organized as follows. In Sec. II, we discuss the inclusion of dipole moments in 3D KMC simulations. Section III contains an overview of the 


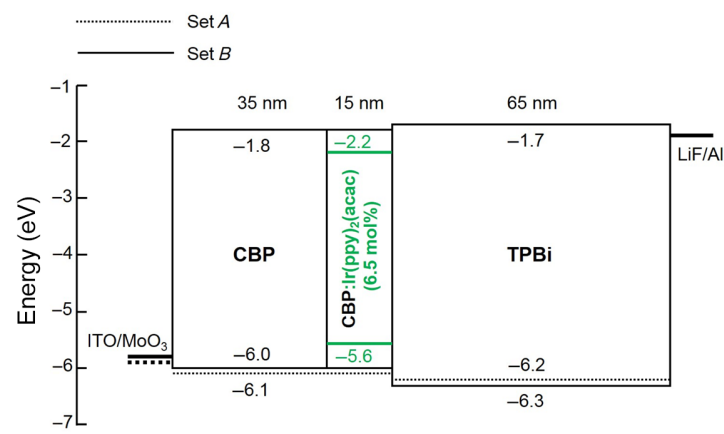

FIG. 2. Energy-level and layer structure of the phosphorescent OLEDs studied. The CBP:Ir(ppy $)_{2}($ acac $)(6.5 \mathrm{~mol} \%)$ emissive layer is sandwiched in between a CBP hole transport layer and a TPBi electron transport layer. $\operatorname{ITO}(70 \mathrm{~nm}) / \mathrm{MoO}_{3}(1 \mathrm{~nm})$ and $\operatorname{LiF}(0.7 \mathrm{~nm}) / \mathrm{Al}(100 \mathrm{~nm})$ are used as anode and cathode layers, respectively. The dotted (full) lines give the energy sets $A$ and $B$, respectively. In this work, 3D KMC simulations are shown to yield a fair description of the current voltage curves and emission profiles reported in Ref. [34] when using set $B$ and including dipole moments on TPBi, or when using set $A$, neglecting the dipole moments on TPBi and assuming a strongly reduced electron hopping attempt rate (see Sec. V).

available experimental and theoretical information about the dipole-moment orientation in TPBi, including data from the literature and from ultraviolet photoelectron spectroscopy (UPS) measurements of TPBi thin films with variable layer thicknesses. The result from UPS measurements of the GSP effect is consistent with the findings from earlier literature results. In Sec. IV, an overview is given of the 3D KMC simulation approach. Section V gives the results, obtained from 3D KMC simulations that neglect and include the effect of the TPBi dipole moments. A comparison is given of the experimental $J(V)$ characteristics, the EQE roll-off curve and the emission profiles, and the sensitivity to the dipole moment size and the net dipole moment orientation is studied. In Sec. $\mathrm{V}$ we summarize the results and conclusions. In the Appendix we analyze using 3D KMC simulations with which accuracy the emission profile can be deduced from the sense layer experiments that were done in Ref. [34].

\section{DIPOLE MOMENTS IN 3D KMC SIMULATIONS}

\section{A. Field due to a dipole in between two metallic electrodes}

The simulations are carried out using the 3D KMC simulation tool Bumblebee [37], that has been described in various earlier publications [20,22,38], after extending the method to include the electrostatic field distribution due to static molecular dipole moments. In the simulations, the molecular sites are located on the grid points of a simple cubic lattice, with a distance $a=1 \mathrm{~nm}$ that represents to a good approximation the average intermolecular distance. The charges are treated using the point-charge approximation and the dipoles are included by making the point-dipole approximation.

The electrostatic field around each dipole is calculated in the following way. We consider a system with a distance $L$ between the interfaces with the two metallic electrode planes at $x_{1}=-L / 2$ and $x_{2}=L / 2$. The dipole is located at a position $\vec{r}_{0}=\left(r_{0, x}, r_{0, y}, r_{0, z}\right)$. Using the image-charge method, the electrostatic potential around an electric charge $Q$ at a position $\vec{r}=\left(r_{x}, r_{y}, r_{z}\right)$ is given by [39]

$$
\begin{aligned}
\phi_{\mathrm{ch}}\left(\vec{r}, \vec{r}_{0}, Q\right)= & \frac{Q}{4 \pi \varepsilon} \sum_{n=-\infty}^{\infty}\left(\frac{1}{\left[\left(r_{x}-r_{x, 0}+2 n L\right)^{2}+\left(r_{y}-r_{0, y}\right)^{2}+\left(r_{z}-r_{0, z}\right)^{2}\right]^{1 / 2}}\right. \\
& \left.-\frac{1}{\left\{\left[r_{x}+r_{0, x}+(2 n+1) L\right]^{2}+\left(r_{y}-r_{0, y}\right)^{2}+\left(r_{z}-r_{0, z}\right)^{2}\right\}^{1 / 2}}\right)
\end{aligned}
$$

with $\varepsilon=\varepsilon_{0} \varepsilon_{r}$ the product of the vacuum permittivity and the relative permittivity. The summation includes the direct Coulomb potential (the $n=0$ term) and contributions due to image charges at virtual locations that are obtained by an alternating reflection in the left and right electrode. The sign of the image-charge contributions alternates upon each reflection.

The electrostatic potential around a point dipole $\vec{p}=\left(p_{x}, p_{y}, p_{z}\right)$ at the position $\vec{r}_{0}$ is given by

$$
\phi_{\mathrm{dip}}\left(\vec{r}, \vec{r}_{0}, \vec{p}\right)=\lim _{Q \rightarrow \infty}\left[\phi_{\mathrm{ch}}\left(\vec{r}, \vec{r}_{0}+\frac{\vec{p}}{2 Q}, Q\right)-\phi_{\mathrm{ch}}\left(\vec{r}, \vec{r}_{0}-\frac{\vec{p}}{2 Q}, Q\right)\right]
$$


Combining Eqs. (1) and (2) leads to

$$
\begin{aligned}
\phi_{\text {dip }}\left(\vec{r}, \vec{r}_{0}, \vec{p}\right)= & \frac{1}{4 \pi \varepsilon} \sum_{n=-\infty}^{\infty}\left(\frac{p_{x}\left(2 n L+r_{x}-r_{0, x}\right)+p_{y}\left(r_{y}-r_{0, y}\right)+p_{z}\left(r_{z}-r_{0, z}\right)}{\left[\left(r_{x}-r_{0, x}+2 n L\right)^{2}+\left(r_{y}-r_{0, y}\right)^{2}+\left(r_{z}-r_{0, z}\right)^{2}\right]^{3 / 2}}\right. \\
& \left.+\frac{p_{x}\left(L+2 n L+r_{x}+r_{0, x}\right)-p_{y}\left(r_{y}-r_{0, y}\right)-p_{z}\left(r_{z}-r_{0, z}\right)}{\left\{\left[r_{x}+r_{0, x}+(2 n+1) L\right]^{2}+\left(r_{y}-r_{0}, y\right)^{2}+\left(r_{z}-r_{0, z}\right)^{2}\right\}^{3 / 2}}\right) .
\end{aligned}
$$

\section{B. Dipole-moment distribution}

The dipole-moment distribution can be included at various levels of refinement. In our simulations, the dipole moments on each TPBi site have a fixed size and are taken randomly from an aspherical orientation distribution with a net preferential orientation that is consistent with results from quantum-chemical simulations [12]. We do not include a possible orientation correlation between the dipole moments of nearly molecules, such as found for some Ir-based emitter molecules in Ref. [36]. If needed, a possible distribution of dipole-moment sizes could be easily added.

The dipole-moment vectors are determined by an azimuthal angle $\varphi$ and a polar angle $\theta$ that is defined as the angle of the dipole moment with respect to the film growth direction (positive $x$ direction). For evaporation deposition on a rotating substrate, no azimuthal dependence of the dipole-vector orientation is expected. We therefore obtain the $y$ and $z$ components of the dipole vector by assuming a random value of the azimuthal angle. For a fully random orientation distribution, the normalized orientation probability distribution function $P(\cos \theta)$ is equal to $1 / 2$ [dashed line in Figs. 1(b) and 1(c)]. A random value of the polar angle can then be obtained by drawing a random number $-1 \leq w \leq 1$, so that $\cos \theta=w$. The spontaneous orientation polarization is growth induced, so that the polarization is directed either parallel or antiparallel to the growth direction. The effect of a such a net dipole-moment orientation can be included by taking $\cos \theta=w+\delta\left(1-w^{2}\right)$. Here $|\delta| \leq 1 / 2$ is a parameter that determines the net preferential orientation, which is characterized by the order parameter

$$
S_{1} \equiv\langle\cos \theta\rangle=\frac{2}{3} \delta
$$

Asphericity of the orientation distribution can be described by taking $\cos \theta=\alpha w /\left[\alpha^{2} w^{2}+\left(1-w^{2}\right)\right]^{1 / 2}$, with $\alpha>0$ a parameter that determines the asphericity of the distribution function, which is characterized by the order parameter

$$
S_{2} \equiv \frac{1}{2}\left(3\left\langle\cos ^{2} \theta\right\rangle-1\right) \cong \frac{1}{5}\left(\alpha^{2}-1\right) .
$$

The approximation is good when $\alpha$ is close to 1 . When both effects are present, these two approaches are combined by taking $\cos \theta=\alpha w_{1} /\left[\alpha^{2} w_{1}^{2}+\left(1-w_{1}^{2}\right)\right]^{1 / 2}$ with $w_{1}=$ $w+\delta\left(1-w^{2}\right)$. If needed, this description of anisotropy can be straightforwardly generalized by using a set of three asphericity parameters $\{\alpha, \beta, \gamma\}$ that also describes an azimuthal dependence of the distribution function.

We find that the method that is given above for obtaining random dipole-moment vectors that are distributed such that $S_{1}$ and $S_{2}$ deviate from zero can provide fair descriptions of the theoretical distribution functions for various materials [12], as shown below for the case of TBPi. However, we cannot exclude that for some materials a more general approach is needed. For any arbitrary dipoleorientation distribution function $P(\cos \theta)$ a set of random values of $\cos (\theta)$ that is consistent with this function can be obtained in the following way. First, the known (measured or calculated) function $P(\cos \theta)$ is used to calculate the cumulative distribution function

$$
u\left(\cos \theta_{0}\right) \equiv \int_{-1}^{\cos \theta_{0}} P(\cos \theta) d(\cos \theta) .
$$

Subsequently, the inverse function $v(u)$ (defined on the interval $[0,1]$ and with function values in the interval $[-1,1])$ is calculated. Drawing random numbers $0 \leq u \leq$ 1 yields then random values of $\cos \theta \equiv v(u)$ with the correct probability distribution.

\section{DIPOLE-ORIENTATION DISTRIBUTION IN TPBI}

\section{A. Overview of theoretical and experimental literature data}

TPBi is a molecule that consists of a phenyl core to which three identical subgroups are attached [Fig. 1(a)]. For gas-phase molecules significantly different theoretical static dipole moments have been reported by several groups, ranging from $2.0 \mathrm{D}$ [11] and $5.5 \mathrm{D}$ [40] to 6.4 and $7.6 \mathrm{D}$ [41]. In the latter study two conformers, in which the three subgroups are symmetrically equivalent or not, were distinguished. The size variation of the theoretical gas-phase dipole moments indicates a strong sensitivity to the computational method. Friederich and co-workers have modeled the thin-film deposition process, 
and found a relatively wide distribution of dipole-moment sizes: $7.0 \pm 3.4 \mathrm{D}$ [12]. The authors furthermore analyzed the dipole-orientation distribution, which is shown in Fig. 1(b). We find that the parametrization scheme that is presented in Sec. II B provides an excellent description of these simulation results, with $\alpha=1.5$ and $\delta=0.068$. The resulting orientation distribution is shown in Fig. 1(c). From a numerical calculation that includes the asphericity $(\alpha \neq 1)$, the corresponding value of $\langle\cos \theta\rangle$ is found to be $S_{1}=0.052$. This is slightly larger than the value of $S_{1}=(2 / 3) \delta \approx 0.045$ that would be obtained from Eq. (3) for $\alpha=1$.

The average dipole-moment orientation can be deduced from a GSP measurement as a function of the layer thickness. When the orientation distribution is uniform across the layer thickness, $\langle\cos \theta\rangle$ follows from the measured layer-thickness-independent internal field $F_{0}$ ("GSP slope") using

$$
\langle\cos \theta\rangle=\frac{\varepsilon_{0} \varepsilon_{r} F_{0} V}{p}
$$

with $V$ the average volume per molecule. For TPBi, Kelvin probe (KP) methods $[11,15,42-45]$ and displacement current measurements (DCM) [11,17] have yielded values of the GSP slope in the range $36-88 \mathrm{~V} / \mathrm{nm}$. Using the theoretical average values $p=7.0 \mathrm{D}$ and $V=0.96 \mathrm{~nm}^{3}$ [12] and the measured value $\varepsilon_{r}=2.89$ [11], Eq. (7) yields then a value of $\langle\cos \theta\rangle$ in the range 0.035-0.092. Recent interest in the application of TPBi in electrets for vibrationbased power generation has stimulated carrying out more detailed studies of the GSP effect. The GSP slope is relatively stable under visible-light illumination and under exposure to atmospheric conditions [45], but depends strongly on the substrate temperature during deposition and the deposition rate. Substrate heating during the deposition to temperatures above room temperature has been argued to give rise to a reduced orientational order of the molecules [17], which vanishes for substrate temperatures of approximately $0.9 T_{g}$, where $T_{g}$ is the glass temperature $\left(397 \mathrm{~K}\right.$, i.e., $124^{\circ} \mathrm{C}$, for TPBi). For deposition at room temperature, the GSP slope was found from a KP study to increase from 36 to $65 \mathrm{mV} / \mathrm{nm}$ for rates that increase from 0.02 to $0.4 \mathrm{~nm} / \mathrm{s}$ [44].

\section{B. UPS experiments}

The GSP slope can also be determined using ultraviolet photoelectron spectroscopy (UPS) experiments [10], viz. from a measurement of the layer thickness dependence of the work function of organic semiconductor thin films that have been deposited on a metallic substrate [10]. We perform such experiments using a He-I $(21.2 \mathrm{eV})$ gas-discharge source for $0-25-\mathrm{nm}$ TPBi layers on a $\mathrm{Au}$ thin-film substrate, evaporation deposited in situ on a substrate at room temperature with a rate of $0.01 \mathrm{~nm} / \mathrm{s}$. As

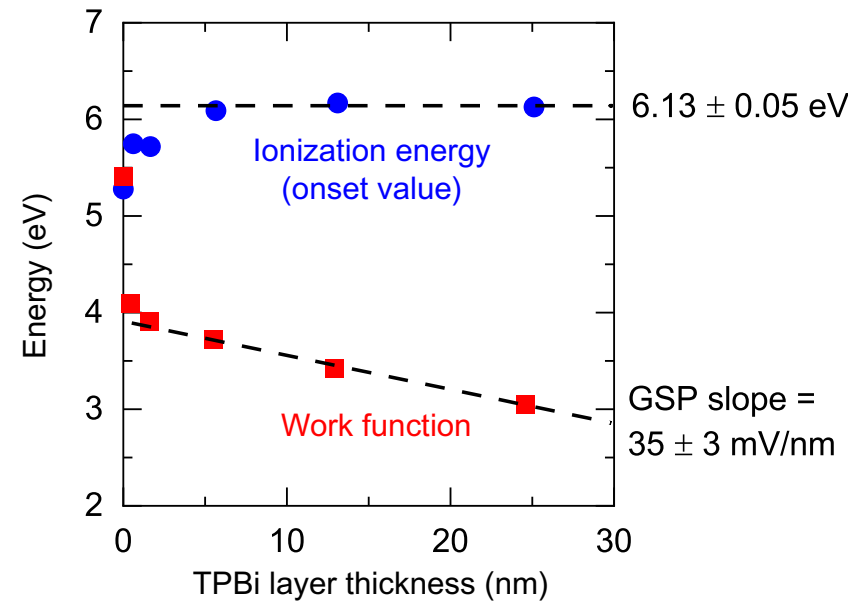

FIG. 3. TPBi-layer-thickness dependence of the TPBi ionization energy and the work function, obtained from UPS experiments of evaporation-deposited films of TPBi on a Au substrate.

shown by Fig. 3, the experiments reveal for sufficiently thick films an almost layer-thickness-independent value of the spectral onset energy of $E_{\text {onset }}=6.13 \pm 0.05 \mathrm{eV}$. This value is determined by extrapolating the tangent through the inflection point at the high-kinetic energy spectral edge to the background intensity level. We note that this energy can in general still differ by $0.1-0.2 \mathrm{eV}$ from the bulk adiabatic ionization energy that is needed in $3 \mathrm{D} \mathrm{KMC}$ simulations, because of the roles of peak broadening due to energetic disorder, the enhanced ionization energy at the vacuum surface, and hole-vibrational coupling [46].

The figure also shows that already when covering $\mathrm{Au}$ with a monolayer of TPBi, the work function decreases from about $5.4 \mathrm{eV}$ by more than $1 \mathrm{eV}$. We attribute that to the inward shift of the Au electron density due to an exchange repulsion interaction with the orbitals of the TPBi overlayer molecules ("pillow effect" or "cushion effect" $[47,48])$. The hole injection barrier is therefore about $2 \mathrm{eV}$ or more, so that hole transfer to the organic layer and a resulting "band bending" is expected to be minimal. The experiments still show a weak indication of hole transfer within the first $2 \mathrm{~nm}$. That might be attributed to enhanced energetic disorder near the interface, possibly in part due to the molecular dipole moments of TPBi and their image-charge interaction with the substrate [49], and to the dipole orientation of TPBi, which gives rise to a net negative surface charge in the first molecular layer that will attract holes from the metal electrode.

For thicknesses above $5 \mathrm{~nm}$, the decrease with increasing thickness is linear, resulting in a work-function slope of $-35 \mathrm{meV} / \mathrm{nm}$ (GSP slope of $35 \mathrm{mV} / \mathrm{nm}$ ). This is close to the value of $36 \mathrm{mV} / \mathrm{nm}$ that has been obtained from KP experiments (see the discussion in Sec. III A) for a similarly small deposition rate $(0.02 \mathrm{~nm} / \mathrm{s})$. Using Eq. (7), with the values of $p, V$, and $\varepsilon_{r}$ that were used in Sec. III A, this slope corresponds to $\langle\cos \theta\rangle \cong 0.036$. Although we 
did not observe any evidence of charging, caused by the photoemission experiment or as a result of visible-light illumination, it cannot be excluded that the measurements underestimate the intrinsic GSP slope due to accumulation of electrons at the vacuum interface. In view of such uncertainties in experiments that probe the GSP slope, the dependence of the GSP slope on the deposition conditions, and the dependence of the order parameter on the average dipole moment that is assumed when using Eq. (7), we analyze in Sec. V D the performance of the device shown in Fig. 1 for various values of $\langle\cos \theta\rangle$.

\section{3D KMC DEVICE SIMULATIONS-METHOD}

Within the 3D KMC simulations, the molecules are located on a simple cubic lattice with a lattice parameter $a=1 \mathrm{~nm}$, corresponding to a total molecular density $N_{t}=10^{27} \mathrm{~m}^{-3}$. As in earlier studies [22,26], electron and hole hopping is described as a Miller-Abrahams-type process with a rate that depends on the hopping distance $R$ and the energy difference $\Delta E$ between the final and initial state as $v=v_{1} \exp [-2(R-a) / \lambda] \exp [-(\Delta E+$ $\left.|\Delta E|) /\left(2 k_{B} T\right)\right]$, with $v_{1}$ the hopping rate to a first nearestneighbor molecule when $\Delta E=0, \lambda$ the wave-function decay length, $k_{B}$ the Boltzmann constant, and $T$ the temperature. Following these earlier studies, we use $\lambda=0.3 \mathrm{~nm}$ and, for hole transport, $v_{1, h}=3.3 \times 10^{10} \mathrm{~s}^{-1}$. All simulations are carried out for $T=300 \mathrm{~K}$.

Figure 2 shows the frontier-orbital energies that are used. For simulations without dipole moments (Sec. V A), we use the values that are indicated as "set $A$." The disorder-averaged HOMO energies, $E_{\mathrm{HOMO}}$, are then as given in Ref. [34], apart from a small $(0.1 \mathrm{eV})$ downward shift of the HOMO energy of $\operatorname{Ir}(\mathrm{ppy})_{2}$ (acac). These values are representative of UPS results that have been obtained for CBP, $\operatorname{Ir}(\mathrm{ppy})_{2}(\mathrm{acac})$ and TPBi by various groups. For the materials used, accurate experimental values for the disorder-averaged LUMO energy, $E_{\mathrm{LUMO}}$, are not available. For $\mathrm{CBP}$ and $\mathrm{TPBi}$, we estimate $E_{\mathrm{LuMO}}$ by adding the measured experimental singlet energy (approximately 3.3 and $3.5 \mathrm{eV}$, respectively) and an estimated singlet exciton binding energy of $1.0 \mathrm{eV}$ to $E_{\mathrm{HOMO}}$. For $\operatorname{Ir}(\mathrm{ppy})_{2}(\mathrm{acac})$ we add the experimental triplet energy $(2.3 \mathrm{eV})$ and an estimated triplet binding energy of $1.1 \mathrm{eV}$ to $E_{\text {номо. Such }}$ relatively large values of the exciton binding energy are consistent with theoretical [50] and experimental [51] studies, which show that the exciton binding energy for smallmolecule organic semiconductors is typically in the range $0.5-1.5 \mathrm{eV}$. A second set of simulations, for systems with TPBi dipole moments, is carried out using slightly adapted HOMO energies ("set $B$ " in Fig. 2; see the motivation for this adaptation in Sec. V B).

In the absence of a dipolar contribution, the frontier orbital energies are assumed to be taken from a spatially uncorrelated Gaussian distribution function with a width (standard deviation) of $0.1 \mathrm{eV}$. From previous simulation and experimental studies, that width is found to be a fair typical value for many systems [20,22,52]. For this parameter value combination, the mobility at a very small carrier concentration $\left(10^{-5}\right.$ charges per molecule) is from $3 \mathrm{D} \mathrm{KMC} \mathrm{simulations} \mathrm{close} \mathrm{to} 2 \times 10^{-9} \mathrm{~m}^{2} /(\mathrm{Vs})$ in the zero-electric field limit, and a factor of approximately 10 larger for a field of $10^{7} \mathrm{~V} / \mathrm{m}$. The mobility increases also with increasing carrier concentration, approximately in a manner as described within the extended Gaussian disorder model (EGDM [53]). The electron mobility is often found to be smaller than the hole mobility, most likely as a result of inavoidable extrinsic trap states [20,54]. For electrons, we consider $v_{1, e}$ therefore as a free parameter, which is adapted to obtain an optimized agreement between the simulation results and the experimental emission profiles.

The dipole-moment contribution to the electrostatic potential at a certain molecule is calculated by summing the contributions from all other dipoles, given by Eq. (3). Screening by the metallic electrodes leads for lateral distances much larger than the device thickness to an exponential decrease of the electrostatic potential around a charge, with an (1/e) decay length of the order $L / \pi$ [39]. One may expect a similar or even faster decay when the field due to a dipole is considered. We include all contributions up to a lateral distance of $\pm 575 \mathrm{~nm}$ (i.e., \pm 5 times the device thickness). The total number of terms in the summation of Eq. (3) is limited to 100.

The excitonic processes in the emissive layer are included in the following manner. The $\operatorname{Ir}(\mathrm{ppy})_{2}(\mathrm{acac})$ exciton energies are assumed to be Gaussian distributed with a width of $0.05 \mathrm{eV}$. The triplet exciton radiative and nonradiative decay rates on $\operatorname{Ir}(\mathrm{ppy})_{2}(\mathrm{acac})$ are $\Gamma_{T, r}=1.14 \times$ $10^{6} \mathrm{~s}^{-1}$ and $\Gamma_{\mathrm{nr}}=5.6 \times 10^{4} \mathrm{~s}^{-1}$, respectively [34]. All excitons that are formed on the emitter molecules are assumed to be a triplet. Triplet exciton transfer between the $\operatorname{Ir}(\mathrm{ppy})_{2}$ (acac) molecules is described as a sum of a Förster-type and a Dexter-type contribution, as detailed in Ref. [22], with a Förster radius $R_{F, \text { diff }}$ of $1.5 \mathrm{~nm}$, a Dexter prefactor (rate in the limit of zero distance) of $k_{D, 0}=$ $2.5 \times 10^{10} \mathrm{~s}^{-1}$, and an exciton wave-function decay length of $\lambda_{\text {exc }}=0.3 \mathrm{~nm}$. For the $6.5 \mathrm{~mol} \%$ CBP: $\operatorname{Ir}(\text { ppy })_{2}$ (acac) systems studied, Förster transfer is the most important process [55]. The Förster radius is close to the value of 1.6 $\mathrm{nm}$ that follows from a recent quantum-chemical calculation study [40]. Triplet quenching with electron or hole polarons (TPQ) on other emitter molecules and triplettriplet annihilation (TTA) involving two triplets on an emitter molecule are also described as a Förster-type process, with for both interactions a Förster radius of $3.5 \mathrm{~nm}$. In earlier 3D KMC studies, this has been argued to be a realistic value [21,24].

The high triplet energies of the CBP and TPBi layers (2.5-2.6 and 2.7-2.8 eV, respectively), prevent triplet deconfinement from the EML to the these layers. 
Furthermore, the simulations and experimental results in Ref. [34] show that essentially no leakage of electrons to the CBP hole transport layer occurs. However, there is some leakage of holes from the EML to the TPBi layer, in particular at large current densities. Experimentally, this is evident from weak emission at wavelengths below $450 \mathrm{~nm}$, with a relative intensity of about $0.1 \%-1 \%$ with respect to the $\operatorname{Ir}(\mathrm{ppy})_{2}$ (acac) peak intensity at about $515 \mathrm{~nm}$. The efficiency loss in the TPBi layer depends on the singlet radiative decay efficiency in the TPBi layer, the fraction of singlets formed, field-induced dissociation, the fraction of holes that reaches the cathode without contributing to exciton formation, bimolecular loss processes, and the position-dependent light-outcoupling efficiency. In view of our focus on the effect of including the TPBi dipoles on the emission from the EML, we do not make

TABLE I. Overview of the simulation parameters used. The frontier orbital energy levels are given in Fig. 2. The approach that is used to include exciton formation and quenching in the CBP and TPBi layers is described in Sec. IV. The range of possible charge-carrier hops and of excitonic interaction processes is limited to 2 and $4 \mathrm{~nm}$, respectively.

\begin{tabular}{|c|c|c|}
\hline Parameter & Description & Value \\
\hline$N_{t}$ & Site density & $10^{27} \mathrm{~m}^{-3}$ \\
\hline$v_{1, h}$ & Hole-hopping attempt rate & $3.3 \times 10^{10} \mathrm{~s}^{-1}$ \\
\hline$v_{1, e}$ & Electron-hopping attempt rate ${ }^{a}$ & $3.3 \times 10^{8} \mathrm{~s}^{-1}$ \\
\hline$v_{1, e}$ & Electron-hopping attempt rate ${ }^{b}$ & $1.0 \times 10^{10} \mathrm{~s}^{-1}$ \\
\hline$\lambda$ & $\begin{array}{l}\text { Electronic wave-function decay } \\
\text { length (holes, electrons) }\end{array}$ & $0.3 \mathrm{~nm}$ \\
\hline$\sigma$ & Electronic disorder energy & $0.10 \mathrm{eV}$ \\
\hline$\lambda_{S, T}$ & $\begin{array}{l}\text { Excitonic wave-function decay } \\
\text { length (singlets, triplets) }\end{array}$ & $0.3 \mathrm{~nm}$ \\
\hline$\sigma_{S, T}$ & Excitonic disorder energy & $0.05 \mathrm{eV}$ \\
\hline$\varepsilon_{r}$ & Relative permittivity & 3.0 \\
\hline$k_{D}$ & Dexter prefactor & $2.5 \times 10^{10} \mathrm{~s}^{-1}$ \\
\hline$T$ & Temperature & $300 \mathrm{~K}$ \\
\hline \multicolumn{3}{|c|}{$\operatorname{Ir}(\mathrm{ppy})_{2}(\mathrm{acac})$} \\
\hline$E_{T, b}$ & Triplet exciton binding energy & $1.1 \mathrm{eV}$ \\
\hline$\Gamma_{T, r}$ & Triplet radiative decay rate & $1.14 \mu \mathrm{s}^{-1}$ \\
\hline$\Gamma_{T, n r}$ & Triplet nonradiative decay rate & $0.056 \mu \mathrm{s}^{-1}$ \\
\hline$R_{F, \text { diff }}$ & Förster radius for exciton transfer & $1.5 \mathrm{~nm}$ \\
\hline$R_{F, \mathrm{TPQ}}$ & Förster radius for $\mathrm{TPQ}^{\mathrm{c}}$ & $3.5 \mathrm{~nm}$ \\
\hline$R_{F, \mathrm{TTA}}$ & Förster radius for TTA ${ }^{\mathrm{d}}$ & $3.5 \mathrm{~nm}$ \\
\hline \multicolumn{3}{|c|}{ TPBi } \\
\hline$p$ & Static dipole moment $\mathrm{e}^{\mathrm{e}}$ & $7 \mathrm{D}$ \\
\hline$\delta$ & Dipole orientation parameter ${ }^{f}$ & 0.068 \\
\hline$\alpha$ & Dipole distribution asphericity & 1.5 \\
\hline
\end{tabular}

${ }^{\text {a}}$ For simulations using energy-level set $A$.

${ }^{\mathrm{b}}$ For simulations using energy-level set $B$.

${ }^{\mathrm{c}}$ TPQ with a hole or electron on another emitter molecule.

dTTA with a triplet on another emitter molecule.

${ }^{\mathrm{e}}$ The effect of a variation is shown in Fig. 5.

${ }^{\mathrm{f}}$ The effect of a variation is shown in Figs. 6 and 7 . an attempt to accurately describe all factors that determine the small emission from the TPBi layer. Instead, the 3D KMC simulations just assume a typical set of simulation conditions: a singlet fraction of $25 \%, \Gamma_{S(T), r}=$ $1(0) \times 10^{8} \mathrm{~s}^{-1}, \Gamma_{S(T), n r}=0(1) \times 10^{5} \mathrm{~s}^{-1}$, and Dexter-type bimolecular loss rates with a similar rate as assumed for excitons on $\operatorname{Ir}(\mathrm{ppy})_{2}$ (acac) (see above). For completeness, the same parameter set is used for describing the excitonics in the CBP layer. The precise values of these parameters are not expected to have a significant effect on the current density, the EQE roll-off, and the emission profile.

The relative permittivity is assumed to be equal to $\epsilon_{r}=3$. Charge-carrier injection from the electrodes is modeled as a Miller-Abrahams-type hopping process with an additional energy barrier, as described in Ref. [56]. At both electrode interfaces a $0.2-\mathrm{eV}$ injection barrier is assumed. Such a small barrier leads to an Ohmic contact. All results are obtained using simulation boxes with a lateral size of $50 \times 50 \mathrm{~nm}^{2}(50 \times 50$ sites $)$ and with a size of $105 \mathrm{~nm}$ (105 sites) in the layer-thickness direction, and using periodic boundary conditions. The final results are an average over typically five-ten boxes, with different random disorder configurations, depending on the box-tobox variation of the simulation results. An overview of all simulation parameters is given in Table I.

\section{3D KMC DEVICE SIMULATIONS-RESULTS}

In this section the effect of introducing static dipole moments at the TPBi sites is studied using 3D KMC simulations. For reference purposes, first simulations within which the dipole moments are neglected are presented (subsection A). Subsequently, results are presented for devices with TPBi dipole moments of $7 \mathrm{D}$, the value that was obtained from theoretical work in Ref. [12] (subsection $\mathrm{B}$ ). In subsection $\mathrm{C}$, the sensitivity to the value of the dipole moments is investigated. In subsection D the sensitivity to its net preferential orientation [order parameter $S_{1}$, see Eq. (4)] and the anisotropy of the angular distribution [order parameter $S_{2}$, see Eq. (5)] are discussed. It is shown that after including dipoles a good description of the experimentally available device characteristics can be obtained when using frontier orbital set $B$ (see Fig. 2), combined with an electron hopping attempt rate $v_{1, e}$ to the first nearest-neighbor sites that is similar to the value for holes $\left(v_{1, e} / v_{1, h}=0.3\right)$, but that when neglecting the effect of dipoles the agreement with experiment is still fair when using a strongly reduced value of $v_{1, e}$ (viz. $\left.v_{1, e} / v_{1, h}=0.01\right)$ in combination with a slightly adapted set of frontier orbital energies (set $A$, see Fig. 2). The LUMO orbital intermolecular transfer integrals that would be obtained by analyzing experimental data while neglecting the dipole-induced effect on the charge transport would thus be significantly misjudged. An overview of the main findings is given in Table II. Comments on the extent to 
TABLE II. Overview of the main 3D KMC simulation results and conclusions. Note that the findings are specific to the devices studied. Comments on the extent to which the findings can be generalized are given in Sec. VI. The energy-level sets $A$ and $B$ are shown in Fig. 2. The third column gives the ratio $v_{1, e} / v_{1, h}$ of the assumed electron- and hole-hopping attempt rates to the first nearest-neighbor sites.

\begin{tabular}{|c|c|c|c|c|}
\hline & $\begin{array}{c}\text { Energy } \\
\text { levels }\end{array}$ & $v_{1, e} / v_{1, h}$ & Figures & Main findings \\
\hline $\begin{array}{l}\text { Simulations } \\
\text { without } \\
\text { dipole } \\
\text { moments }\end{array}$ & set $A$ & 0.01 & 4(a),(b),(d) & $\begin{array}{l}\text { Simulations for the nominal (literature-based) set of energy levels }(A) \text { and using a } \\
\text { very small electron-hopping attempt rate }{ }^{\mathrm{a}} \text { provide the following: } \\
\text { - fair agreement with the experimental } J(V) \text { characteristics, but with a slightly } \\
\text { too large slope on a double-log scale. } \\
\text { - good agreement (within approximately } 1 \% \text { ) with the experimental EQE roll-off. } \\
\text { - qualitatively good agreement with the experimental shape of the emission } \\
\text { profile }^{\mathrm{b}} \text {. }\end{array}$ \\
\hline $\begin{array}{l}\text { Simulations } \\
\text { with 7D } \\
\text { dipole } \\
\text { moments }\end{array}$ & set $B$ & 0.3 & 4(a),(b),(e) & $\begin{array}{l}\text { Simulations using a more realistic electron-hopping attempt rate and using a } \\
\text { slightly adapted set of energy levels }(B) \text { provide }{ }^{c} \text { the following: } \\
\text { - excellent agreement with the experimental } J(V) \text { characteristics. } \\
\text { - qualitatively good agreement with the experimental shape of the emission profile } \\
\text { - fair agreement ( } 1-2 \% \text { deviation) with the experimental EQE roll-off. }\end{array}$ \\
\hline $\begin{array}{l}\text { Sensitivity } \\
\text { to the } \\
\text { dipole } \\
\text { moment size }\end{array}$ & set $B$ & 0.3 & 5 & $\begin{array}{l}\text { - Strong increase of the current density when reducing the dipole moment. } \\
\text { - Strong increase of the EQE when reducing the dipole moment. } \\
\text { - The EQE increase is in part due to an improved confinement of holes in the EML. } \\
\text { - Good agreement with the experimental EQE roll-off for approximately } 6.5 \mathrm{D} \\
\text { dipole moments. }\end{array}$ \\
\hline $\begin{array}{l}\text { Sensitivity } \\
\text { to the } \\
\text { dipole } \\
\text { moment } \\
\text { orientation } \\
\text { distribution }\end{array}$ & set $B$ & 0.3 & 6,7 & $\begin{array}{l}\text { - Strong effect of the net dipole-moment orientation on the EQE }\left[S_{1}, \text { Eq. (4)], }\right. \\
\text { due to an effect on the recombination efficiency and the emission profile. } \\
\text { - For the devices studied, with a small }(0.2 \mathrm{eV}) \text { electron injection barrier: } \\
\text { no effect on the ease of electron injection. } \\
\text { - An effect on the electron injection is found for injection barriers exceeding } 0.6 \mathrm{eV} \text {. } \\
\text { - Weak sensitivity to the asphericity of the distribution function }\left[S_{2}, \text { Eq. (5).] }\right.\end{array}$ \\
\hline
\end{tabular}

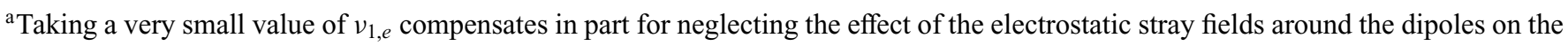
energetic disorder in the TPBi layer, which effectively leads to a reduced electron mobility in that layer.

${ }^{\mathrm{b}}$ The experimental emission profiles were determined in Ref. [34] using the sense-layer method, employing a 0.5-nm layer of Ir-cored red phosphorescent emitters. In the Appendix, these experiments are from 3D KMC simulations shown to provide a fair view on the shape of the profiles, even though the trapping of charges by the sensing molecules and the long-distance exciton transfer to these molecules are found to give rise to differences with the detailed emission profiles from the $\operatorname{Ir}(\mathrm{ppy})_{2}(\mathrm{acac})$ molecules.

${ }^{\mathrm{c}}$ Simulations using set $B$ and $v_{1, e} / v_{1, h}=0.3$, but without dipole moments, show a current density that is a factor of $10-30$ too large [Fig. 4(a)] and an EQE that is approximately 4\% too large [Fig. 4(b)].

which the results that are obtained for the specific device that is studied in this work can be generalized are given in Sec. VI.

\section{A. Simulations without static dipole moments}

We first investigate to what extent 3D KMC simulations can provide a fair description of the device performance, consistent with experimental findings that have been reported in Ref. [34], when the effect of dipole moments on the TPBi molecules is not included. From simulations with varying values of the HOMO and LUMO energies and with a varying value of the hopping attempt rate $v_{1, e}$ for electrons, we find that an overall fair agreement with the measured $J(V)$ curve, roll-off curve, and voltage dependence of the emission profile is obtained when using the dashed energy levels given in Fig. 2 (energy-level set $A$ ) and for $v_{1, e}=10^{-2} \times v_{1, h}$. Apart from the 1.0-eV LUMO orbital shift that is motivated in Sec. IV, the frontier orbital energies differ only weakly (by at most $0.1 \mathrm{eV}$ ) from the values that have been given in Ref. [34]. Such a correction is of the order of the accuracy of the analysis of UPS experiments [46]. All other simulation parameters are as given and motivated in Sec. IV, and remain unchanged when performing simulations including dipole moments (see Sec. V B).

Figure 4(a) shows a comparison of the calculated and measured $J(V)$ curves. On the double-log scale used, the calculated and experimental $J(V)$ curves are almost linear, albeit with slightly different slopes (approximately 5.9 and approximately 6.9 , respectively). The current density that is obtained when neglecting the TPBi dipoles 

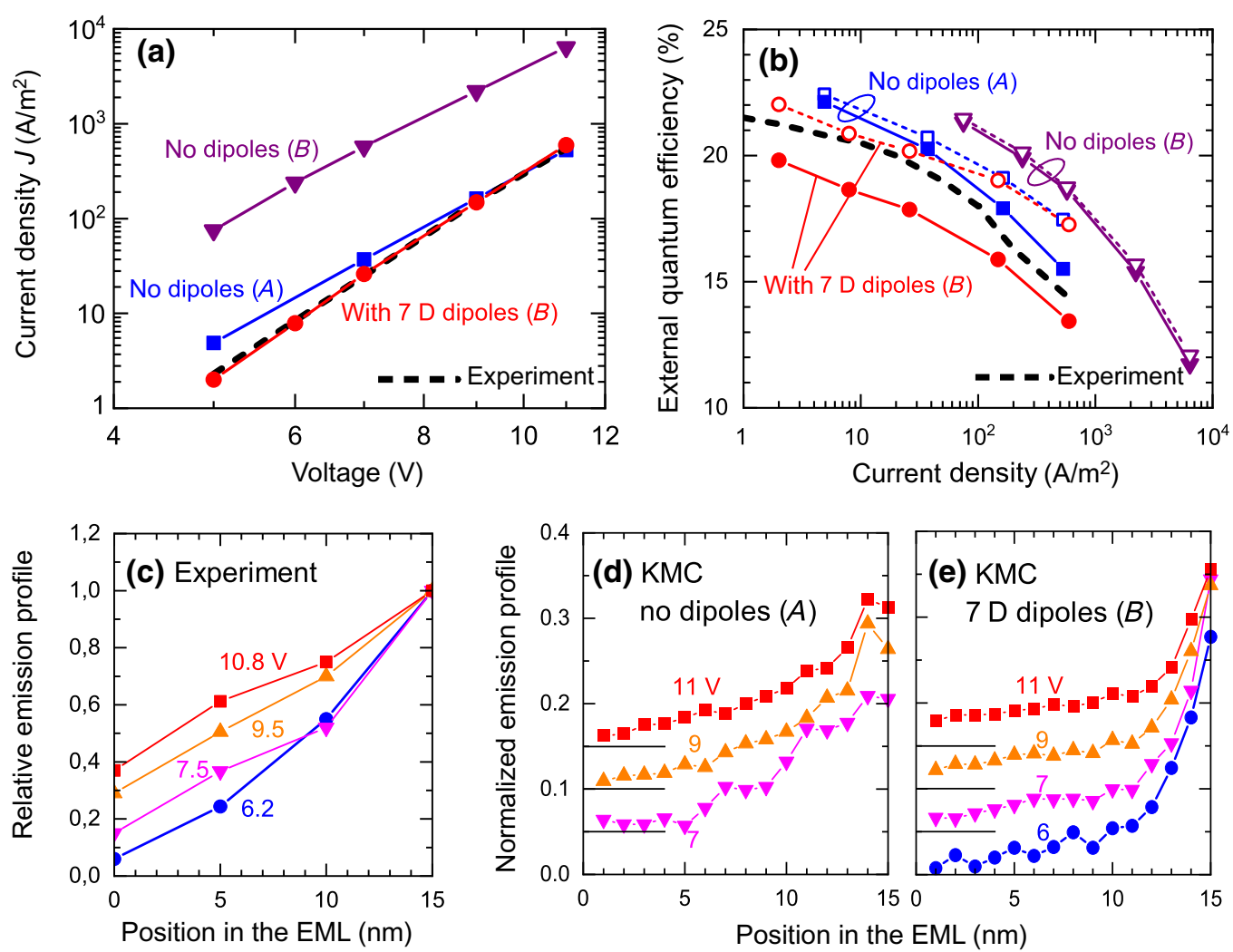

FIG. 4. Comparison of the 3D KMC simulations results for the devices in Fig. 2 with the experimental results of Ref. [34] [thick dashed curves in (a) and (b), and all data in (c)]. The simulations marked $A$ and $B$ use frontier energy-level sets $A$ or $B$ (see Fig. 2) with for all materials $v_{1, e}=0.01 \times v_{1, h}$ and $v_{1, e}=0.3 \times v_{1, h}$, respectively (see Secs. V A and V B). (a) $J(V)$ curves from simulations without and including TPBi dipole moments. (b) Current-density dependence of the external quantum efficiency [ $\eta_{\mathrm{EQE}}$, full curves, see Eq. (8)] and of $\eta_{\text {EQE,EML }}$ [dashed curves, see Eq. (9)]. (c) Emission profiles as a function of the distance to the HTL | EML interface, obtained from sense-layer experiments at the indicated voltages and expressed with respect to the intensity that is obtained using a sense layer at the EML | ETL interface. (d),(e) Normalized emission profiles for various voltages, obtained from 3D KMC simulations neglecting and including the TPBi dipole moments. The 7-, 9-, and $11-\mathrm{V}$ data are for clarity shifted by $0.05,0.10$, and 0.15 .

agrees at high voltages well with experiment, but is at $5 \mathrm{~V}$ approximately a factor of 2 too large. A large slope reflects strong energetic disorder. We attribute the finding of a slightly too small slope to the neglect of dipolar-induced disorder in the TPBi layer, and show in Sec. V B that including the dipoles indeed leads to a $J(V)$ curve with a steeper slope and an essentially perfect agreement with experiment.

Figure 4(b) shows a comparison of the experimental EQE roll-off curve [Fig. 1(d) in Ref. [34], thick dashed black curve] with the calculated curve that is obtained when neglecting any possible emission in the transport layers (full blue curve), i.e.,

$$
\eta_{\mathrm{EQE}}=\eta_{\mathrm{IQE}} \eta_{\mathrm{out}}=\frac{\kappa_{r}}{J / e} \eta_{\mathrm{out}} .
$$

Here, $\eta_{\mathrm{IQE}}$ is the internal quantum efficiency, $\eta_{\text {out }}$ is the light outcoupling efficiency, and $\kappa_{r}$ is the rate of radiative decay processes in the EML per unit area. From an analysis that is shown in Fig. 4(a) of Ref. [34], which includes the effect of the partial orientation of the transition dipole moments of the emitter molecules, it follows that the maximum possible EQE after taking the 95\% PL efficiency of the $\operatorname{Ir}(\mathrm{ppy})_{2}(\mathrm{acac})$ into account is $23.5 \%$. That implies that $\eta_{\text {out }}=24.7 \%$. Figure 4(b) shows that the calculated EQE roll-off curve is similar to the experimental curve, although it is shifted 1 to $1.5 \%$ upward and is less curved than the experimental curve.

In Fig. 4(b) the dashed blue curve gives the EQE that would follow from the radiative decay efficiency in the EML, $\eta_{r, \mathrm{EML}}$ :

$$
\eta_{\mathrm{EQE}, \mathrm{EML}}=\eta_{r, \mathrm{EML}} \eta_{\mathrm{out}}=\frac{\kappa_{r}}{\kappa_{r}+\kappa_{\mathrm{nr}}+\kappa_{\mathrm{TPQ}}+\kappa_{\mathrm{TTA}}} \eta_{\mathrm{out}},
$$

where $\kappa_{\mathrm{nr}}, \kappa_{\mathrm{TPQ}}$, and $\kappa_{\mathrm{TTA}}$ are the rates of nonradiative decay, TPQ and TTA processes per unit of area in the EML, respectively. The difference with the full blue curve, which is the curve that should be compared with experiment, shows that the blocking of holes at the interface with 
the TPBi layer is imperfect, in particular, at high voltages. We note that the $\eta_{\mathrm{EQE}, \mathrm{EML}}(J)$ roll-off curve is not necessarily equal to the roll-off curve that would be obtained in the case of perfect blocking, because of the effects of imperfect blocking on the shapes of the charge and triplet density profiles in the EML.

The normalized emission profiles that are obtained for various voltages are shown in Fig. 4(d). Consistent with the experimental findings [Fig. 4(c)], the simulated emission profiles are at low voltages strongly peaked near the cathode side of the EML. At higher voltages the profiles become somewhat more uniform. The detailed shapes of the experimental and simulated profiles are seen to be somewhat different. For example, the simulated intensity in the $0-5 \mathrm{~nm}$ range seems to be somewhat smaller than as would follow from the sense-layer experiment. However, we find from explicit 3D KMC simulations of the sense layer experiments (see the Appendix) that a somewhat increased relative intensity is expected in this zone, due to hole trapping at the sense molecules. These simulations also show that the sensed intensity near the cathode side of the EML is expected to be somewhat reduced. These effects are expected to happen even when using such a small concentration of sense molecules that their effect on the current density is smaller than typical experimental sample-to-sample variations, as in Ref. [34]. As is shown in the Appendix, quantifying the difference is hampered by a lack of detailed knowledge of the frontier orbital energies of the sense-layer molecules. Irrespective of such details, the sense-layer experiments are still expected to show the strongly unbalanced shape of the emission profile. However, the nonuniformity of the actual emission profile is for this system expected to be underestimated by the sense-layer experiments.

The finding that a very small value of $v_{1, e}$ is needed to obtain an emission profile that is peaked near the cathode interface is qualitatively consistent with the known relatively small electron mobility of TPBi. The time-offlight (TOF) electron mobility of TPBi [57], extrapolated to zero field, is 1 to 2 orders of magnitude smaller than the hole and electron TOF mobilities of CBP [58]. The small electron mobility is, at least in part, due to the dipolemoment-induced disorder of TPBi, which is neglected here. Using a small value of $v_{1, e}$ can only partially compensate for neglecting the effects of the TPBi dipole moments. Firstly, the approach neglects the resulting energetic disorder. Secondly, the effective electron mobility in the EML, which does not contain TPBi, might be underestimated. In the simulations that possible error is most likely compensated by the use of a somewhat too small difference between the CBP (host) and $\operatorname{Ir}\left(\right.$ ppy) ${ }_{2}$ (acac) (guest) LUMO energies. Easier electron transport via the CBP host then enhances the effective electron mobility in the EML. We find indeed that the emission profile is weakly sensitive to $\pm 0.1 \mathrm{eV}$ variations of the $\operatorname{Ir}(\mathrm{ppy})_{2}$ (acac) LUMO energy.
We can also not exclude that the fair agreement of the emission profile is due to a somewhat too large difference between the host and guest HOMO energies, leading to a reduced effective hole mobility in the EML that restores the electron-hole mobility balance.

\section{B. Simulations including static dipole moments}

In the previous subsection, simulations without static dipole moments are shown to provide a fair agreement with the measured current-voltage and efficiency roll-off curves. However, the simulations are carried out using an unrealistically small value of the hopping attempt rate for electrons, viz. a factor of 100 smaller than for hole hopping. We find that an even better agreement with experiment can be obtained when including static dipole moments and when using a hopping attempt rate for electrons that is only a factor of about 3 smaller than for holes $\left(v_{1, e}=0.3 \times v_{1, h}\right)$. The low experimental electron mobility of TPBi is now "automatically" well described by including the dipole-induced disorder. As in earlier 3D KMC simulation studies, $v_{1, e}$ is still somewhat smaller than $v_{1, h}$. The effect may be due to unintentional electron trap states (see also Sec. III A). In this subsection, we study the results of simulations with $7 \mathrm{D}$ TPBi dipole moments, the average theoretical value obtained in Ref. [12], and with a net orientation and anisotropy $(\alpha=1.5$ and $\delta=0.068)$ that follow from the same study. The corresponding orientation order parameter is $S_{1}=\langle\cos \theta\rangle=0.052$ (see Sec. II A). With these dipole moments, the width of the TPBi HOMO and LUMO density of states is increased from 0.10 to $0.187 \mathrm{eV}$. We find that in simulations that are based on frontier orbital set $A$ the increased width of the HOMO density of states leads to a too large efficiency loss, due to very weak hole blocking. Imperfect hole blocking is also evident from the experimental results in Ref. [34], but not to the extent that is predicted using frontier orbital set $A$. We therefore carry out the simulations using the slightly adapted frontier orbital set $B$, with HOMO energies that are still within the experimental uncertainty consistent with the results of UPS studies. This leads to better hole confinement in the EML due to a $0.2 \mathrm{eV}$ larger difference between the HOMO energies of the CBP host and the TPBi electron transport layer. Figure 4 contains the simulation results. In the next subsection, we study the sensitivity to the value of the dipole moment and show that using a value that is approximately $10 \%$ smaller provides an even better agreement with experiment.

The calculated $J(V)$ curve that is obtained with $7 \mathrm{D}$ dipole moments [red curve in Fig. 4(a)] agrees excellently with the experimental result that was reported in Ref. [34]. As anticipated in Sec. V A, including the dipole-induced additional energetic disorder in the TPBi layer leads to a larger slope. We note that the excellent agreement of the theoretical and experimental curves, obtained from an 
approach that is not based on detailed material-specific charge-transport studies, might be somewhat fortuitous and could be a result of compensating errors. We nevertheless regard the set of material parameters that is assumed as representative and as a good starting point for studying the effect of including dipole-moment-induced effects. The figure also shows that for this set of simulation parameters the current density becomes a factor 10-40 larger when switching off the TPBi dipole moments (purple curve).

Figure 4(b) shows that the EQE ( $\eta_{\mathrm{EQE}}$, full red curve) is significantly $(2-4 \%)$ smaller than the value that would follow from the radiative deacy efficiency in the EML ( $\eta_{\mathrm{EQE}, \mathrm{EML}}$, red dashed curve). The difference is caused by an efficiency loss due to imperfect hole blocking at the EML $\mid \mathrm{TPBi}$ interface. At $J=300 \mathrm{~A} / \mathrm{m}^{2}$, the efficiency loss in the EML $\left(1-\eta_{r \text { EML }}\right)$ and the additional IQE loss due to imperfect hole blocking $\left(\eta_{r, \mathrm{EML}}-\eta_{\mathrm{IQE}}\right)$ are from our simulations approximately $22 \%$ and $19 \%$, respectively. From an analysis of the experiments in Ref. [34], these percentages are $21 \%$ and $12 \%$, respectively.

Figure 4(e) shows the normalized emission profiles that are obtained for various voltages. The emission profiles are peaked near the cathode side of the EML, but become somewhat more uniform across the emissive layer thickness with increasing voltage. The precise shape of the roll-off curve is for these devices thus the result of a complex interplay between the increasing role of bimolecular processes with increasing voltage, the changing shape of the emission profile in the EML, and the increasing loss of holes to the TPBi layer. Qualitatively, the emission profiles are consistent with the results from sense-layer experiments, shown in Fig. 4(c). As discussed in the previous subsection, making a more detailed comparison with the experimental emission profiles is hampered by the lack of detailed knowledge of the frontier orbital energies of the sense molecules used, and due to the somewhat distorted shape of the profiles that such experiments are expected to yield.

\section{TPBi dipole-moment variation}

Figure 5 shows the effect of a variation of the TPBi dipole moment $p$ in the range $0-8 \mathrm{D}$, obtained for 3D KMC simulations using energy-level set $B$ at 7,9 , and $11 \mathrm{~V}$. The results that are obtained for $p=0 \mathrm{D}$ are also included in Figs. 4(a) and 4(b) (purple curves). Figure 5(a) shows that for small $p$ the decrease of the 10-log of the current density with $p$ is to a good approximation a second-order effect, as expected for the case of almost randomly oriented dipoles. For $p<2 \mathrm{D}$, the decrease is less than about $20 \%$. In the $4 \mathrm{D}<p<8 \mathrm{D}$ range, the decrease of $\log _{10}(J)$ with increasing $p$ is more close to linear.

Figure 5(b) shows that when completely switching off the dipoles, the EQE increases by approximately $5 \%$. This large increase is caused by an almost perfect hole confinement in the EML, as may be seen from a comparison of the $\eta_{\mathrm{EQE}}$ and $\eta_{\mathrm{EQE}, \mathrm{EML}}$ curves in Fig. 4(b), and by a more uniform emission profile in the EML, as may be seen from Fig. 5(c). As long as $p$ is smaller than approximately $4 \mathrm{D}$, the main effect of the dipole moments is a reduction of the current density and a slight increase of the nonuniformity of the emission profile. For larger values of $p$, the confinement of holes to the EML is no longer perfect, leading to a downward shift of the roll-off curve. The decrease of the EQE is still modest in the range of $4-6 \mathrm{D}$, but is already quite large (about 5\%) in the 6-8 D range. A comparison with the experimental roll-off curve suggests that simulations with $p \cong 6.5 \mathrm{D}$ would be expected to yield an
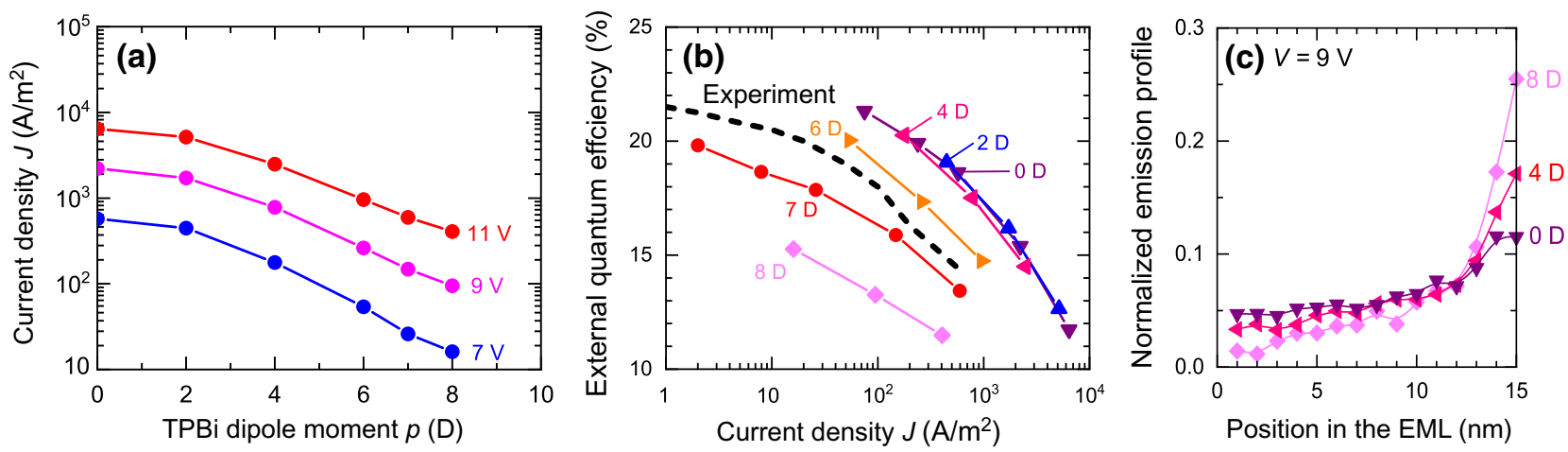

FIG. 5. Results of a 3D KMC simulation study of the sensitivity of the performance of the devices in Fig. 2 (with energy-level set $B$ ) to a variation of the TPBi dipole moment $p$. (a) Dependence of the current density at various values of the voltage on $p$. (b) Roll-off curves $\eta_{\mathrm{EQE}}(J)$ for various values of $p$, obtained from simulations at 7, 9, and $11 \mathrm{~V}$, and (for $p=0$ and $7 \mathrm{D}$ ) also at 5 and $6 \mathrm{~V}$. The labels of each curve are located at the 7-V point. The dashed curve gives the experimental data [34]. (c) Normalized emission profiles in the EML at $9 \mathrm{~V}$, for various values of $p$. Panels (a) and (b) show that the effect on the $J(V)$ characteristics and the EQE roll-off of "switching on" the static dipole moments of the TPBi sites in the ETL, while keeping all other simulation parameters fixed, becomes already significant beyond a value of the molecular dipole moments of approximately 3 D. Panel (c) shows that the resulting reduced effective electron mobility in the ETL strongly affects the shape of the emission profile. 

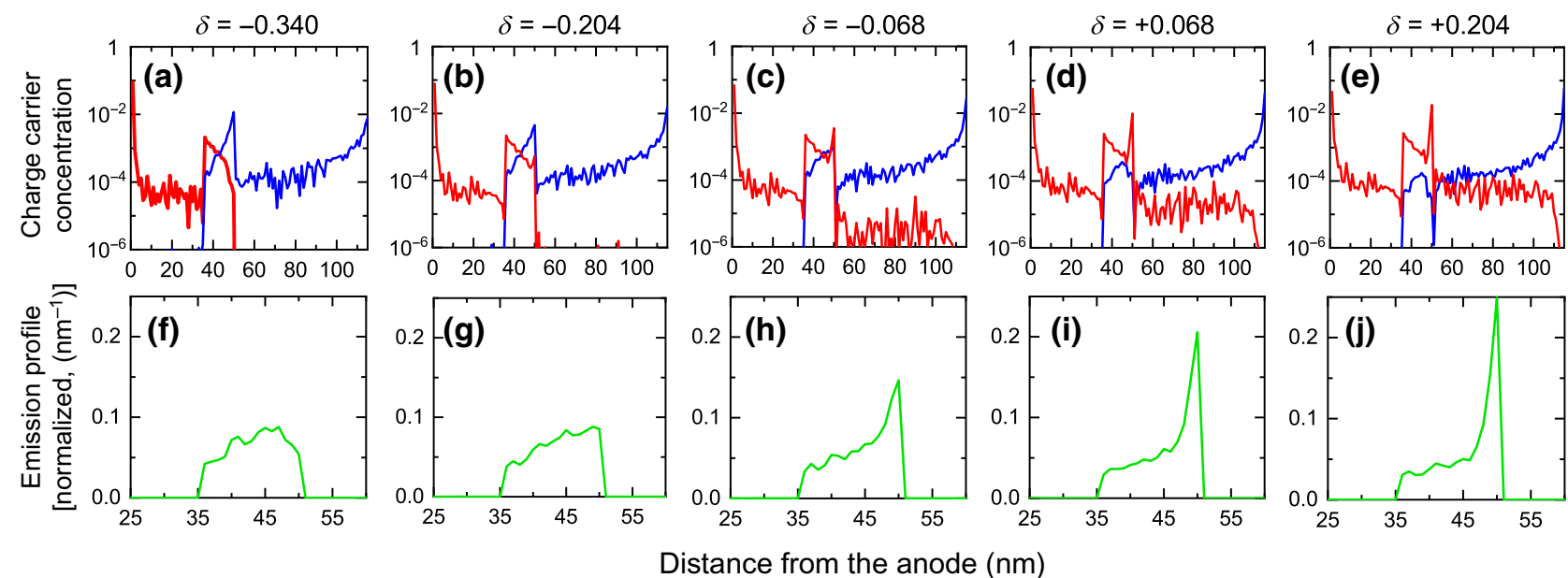

FIG. 6. Charge-carrier concentration profiles [(a)-(e)] and normalized emission profiles [(f)-(j)] across the thickness of the device, obtained from 3D KMC simulations at $V=11 \mathrm{~V}$ for varying values of the dipole-orientation parameter $\delta$ and for a fixed value of the asphericity parameter $\alpha=1.5$. For TPBi, $\delta=+0.068$ (see Sec. III A). In (a)-(e) the red (blue) curves give the hole (electron) concentration. The figure shows that for large positive values of $\delta$ the hole blocking at the EML | TPBi interface becomes nonideal, leading to singlet formation in the TPBi layer that is expected to contribute to an EQE loss due to quenching processes.

excellent agreement with the experimental roll-off curve. Furthermore, also the current density and the shape of the emission profile are then still in good agreement with experiment. We note that the value of $6.5 \mathrm{D}$ is within well within the width of the theoretical distribution of TPBi dipole moments that has been obtained in Ref. [12].

\section{Sensitivity to the dipole-orientation distribution}

We find a strong effect of the TPBi dipole-orientation distribution on the device performance and the IQE, as may be seen from Figs. 6 and 7. The simulation results that are shown in these figures are obtained for $p=7 \mathrm{D}$ and $\alpha=1.5$, using energy-level set $B$. Figures 6(a)-6(e) show for various values of the orientation parameter $\delta$, which determines $S_{1}$, the calculated electron and hole density profiles at $11 \mathrm{~V}$, and Figs. 6(f)-6(j) show the corresponding emission profiles. For strongly positive values of $\delta$, the simulations show that the hole blocking at the EML|TPBi interface is imperfect. The IQE is then found to be reduced due to a reduced recombination efficiency and strong TPQ near EML|TPBi interface due to the large positive space charge at that interface. For strongly negative values of $\delta$, electron accumulation near the EML/TPBi interface is expected to enhance the TPQ loss. Figure 5 gives the IQE at 7 and $11 \mathrm{~V}$ as a function of $\delta$, and shows that these various effects lead to an optimum IQE for slightly negative values of $\delta$. For comparison, the figure also shows the recombination efficiency in the EML $\left[\eta_{r \text {,EML }}\right.$, defined in Eq. (9)]. For positive and small negative values of $\delta$, $\eta_{r \text {,EML }}$ is significantly larger than $\eta_{\mathrm{IQE}}$, as the blocking of holes at the EML/TPBi interface is then imperfect. For the four cases with $\delta \leq 0.068$, the $J(V)$ curves are quite independent of $\delta(<10 \%$ variation $)$. However, the simulations for $\delta=0.204$ show a $30 \%-40 \%$ enhanced current density.

The strong sensitivity of the charge-carrier density and emission profiles to $\delta$, and hence $S_{1}$, that is shown in Fig. 6

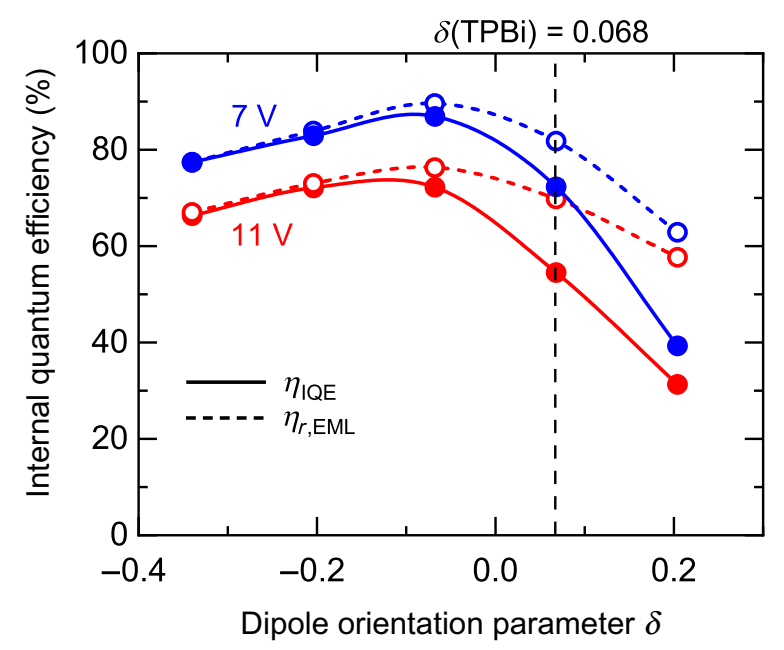

FIG. 7. Dependence of the IQE and the radiative decay efficiency in the EML on the dipole orientation parameter $\delta$, obtained from 3D KMC simulations for the device in Fig. 2 at 7 and $11 \mathrm{~V}$. For TPBi, $\delta=0.068$ (vertical dashed line, see Sec. III A). The simulations were performed for $p=7 \mathrm{D}$ and $\alpha=1.5$, using energy level set $\mathrm{B}$. The closed symbols give $\eta_{\mathrm{IQE}}$ and the open symbols give $\eta_{r \text {,EML }}$ [defined in Eq. (9)]. The curves are guides-to-the-eye. The figure shows that a variation of the net dipole orientation can significantly affect the IQE. For values of $\delta$ for which the difference between the full and dashed curves is large, the IQE is reduced strongly be a nonideal recombination efficiency. Such a loss could be prevented by improving the blocking of holes at the EML|ETL interface. 
may be understood more quantitatively from a consideration of the dependence of the dipole-orientation-induced areal charge density at the EML|TPBi interface on $S_{1}$ :

$$
\sigma_{A}=-\frac{p S_{1}}{a^{3}} .
$$

This leads to an electron energy shift at the interface that is equal to

$$
\Delta E=\frac{x_{\mathrm{int}}\left(L-x_{\mathrm{int}}\right)}{L} \frac{e \sigma_{A}}{\varepsilon_{0} \varepsilon_{r}},
$$

where $x_{\text {int }}$ is the distance of the interface from the anode interface and $L$ is the active layer thickness. For the device studied $\left(S_{1}=0.052\right), \sigma_{A}=-1.2 \times 10^{-3} \mathrm{C} / \mathrm{m}^{2}$, i.e., $7.6 \times 10^{-3}$ electron charges per $\mathrm{nm}^{-2}$, and $\Delta E=1.29 \mathrm{eV}$. Figure 6 shows that the holes in the EML tend to compensate (screen) $\sigma_{A}$. Even at the voltage considered $(11 \mathrm{~V}), \sigma_{A}$ is comparable to the areal charge density due to the injected electrons and holes in the EML. The effect on the charge density profiles and the IQE is therefore significant.

The dipole-moment orientation can also affect the injection of electrons from the cathode into the TPBi electron transport layer. For the energy-level structure used in this Paper, with an electron injection barrier of $\Phi_{e}=0.2 \mathrm{eV}$, such an effect does not yet occur: we find that the effect on the current density of a reversal of $S_{1}$ is very small ( $10 \%$ or less in the $7-11 \mathrm{~V}$ range). Even for $\Phi_{e}=0.6 \mathrm{eV}$, a reversal of $S_{1}$ leads only to a reduction of the current density by approximately $25 \%$. The large dipole-induced disorder facilitates injection into the deep tail states of the LUMO [59], so that the injection process then still does not limit the current density. However, for $\Phi_{e}=1.0 \mathrm{eV}$ we find that the current density in the $7-11 \mathrm{~V}$ range is a factor approximately 60 smaller than for $\Phi_{e}=0.2 \mathrm{eV}$, and that a reversal of $S_{1}$ further reduces the current density by a factor 8-10. The system is then in the injection-limited current regime, for which the dipole-orientation-induced field gradient near the cathode interface facilitates electron injection when $S_{1}$ is positive.

We find that the simulation results are only weakly sensitive to a change of the anisotropy parameter $\alpha$ from $3 / 2$ (the value used throughout this work) to $2 / 3$. The dipole moments are then more preferentially oriented in directions parallel to the film plane. The probability density $P(\cos \theta)$ is then approximately a factor 3 larger for $\cos \theta=0$ than for $\cos \theta= \pm 1$, and $S_{1}=0.038$. In the $5-11 \mathrm{~V}$ range, the current density is $30 \%-50 \%$ enhanced. The width of the LUMO DOS is not affected by this change. We therefore regard the observed increase of the current density as a consequence of a more subtle change of the dipole-induced energy-level landscape.

\section{SUMMARY AND CONCLUSIONS}

We have studied how the presence of large dipole moments of the molecules in the ETL of a prototypical green phosphorescent OLED affects the $J(V)$ characteristics and the EQE roll-off, and to what extent these effects depend on the detailed dipole-moment-orientation distribution. For that purpose, the presence of the dipole moments has been included in the 3D KMC simulations, allowing us to investigate their effect in a fully mechanistic manner. Within the simulations, implications such as the development of a spatially correlated disorder and the additional field due to image dipoles near the metallic electrodes are "automatically" included.

Our study has focussed on an experimentally wellcharacterized device within which TPBi is the electrontransporting material [34]. Advantageously, theoretical and experimental studies of the dipole-moment distribution in TPBi thin films, including the results of a UPS study in this work, provide a consistent picture of the orientation distribution. We find that 3D KMC simulations within which the TPBi dipole moments have been included lead to a $J(V)$ curve, a EQE roll-off and an emission profile that are in good agreement with the experimental data. A key element of the experimental validation was the comparison with the measured emission profile [34], which was obtained using sense-layer experiments. From 3D KMC simulations for devices including a $\delta$-function doping such as used experimentally, we find that the effect of a very thin layer of sense molecules on the current density can be minor, as reported in Ref. [34]. Nevertheless, charge trapping on the sense molecules is expected to slightly distort the shape of the sensed emission profile. Furthermore, the sense-layer experiments are expected to underestimate the emission near the EML | TPBi interface, where strong charge accumulation occurs and where the emission shows a maximum. The effect is, at least in part, due to the relatively large Förster radius for exciton transfer to the sensing molecules, about $2.5 \mathrm{~nm}$ for the system that is studied, combined with the proximity of the ETL, from which no excitons are transferred.

The simulations have allowed various sensitivity studies to be performed. Firstly, we find that switching off the dipole moments leads to a current density that is more than 1 order of magnitude too large, due to the reduced energetic disorder, and an EQE that is significantly larger than as found experimentally. The latter effect is specific to the device that has been studied, and is due to the elimination of deconfinement of holes from the EML to the TPBi layer and to the development of a more uniform emission profile in the EML. Secondly, a variation of the net dipole-moment orientation has shown that its value has a significant effect on the device efficiency, and that for the specific devices studied a reversal of orientation direction would be expected to lead to a relative increase of the 
IQE (and EQE) by $10 \%-20 \%$, depending on the voltage (see Fig. 5). This is mainly due to the improved blocking of holes at the EML | TPBi interface. We note that such a redesign might be feasible in practice. Although many common electron transporting materials show a positive value of the orientation order parameter $S_{1}$ and the giant surface potential [14], it has been shown to be possible to synthesize materials with a negative giant surface potential [60]. Thirdly, an ETL with a net dipole-moment orientation such that $S_{1}>0$ can give rise to improved electron injection, viz. in the presence of such a high electron injection barrier that the transport is in the injection limited current (ILC) regime. However, the injection is already facilitated by the dipole-induced enhanced disorder, so that for TPBi the effect of its dipole orientation is only expected to be significant for very large injection barriers (above about $0.6 \mathrm{eV})$.

We have shown that 3D KMC simulations within which the TPBi dipole moments are neglected can still yield current density and roll-off curves and emission profiles that are in fair agreement with experiment, viz. when (1) using a very small value of $v_{1, e}$ ( 2 orders of magnitude smaller than $v_{1, h}$ ) that partially corrects for the reduced electron mobility that results from the dipole-induced disorder, and (2) with slightly different frontier orbital energies (set $A$ instead of $B$ ). However, simulations that neglect the dipoleinduced disorder do not provide a proper view on the sensitivity to a variation of the hole blocking barrier at the EML|TPBi interface or of a variation of the electron injection barrier at the cathode interface.

The methodology that has been developed in this Paper has been applied to only one case, leading to devicespecific conclusions concerning the effects of dipoles. However, we regard the approach as a good starting point for introducing the effects of dipoles in 3D KMC simulations of any general OLED device. As discussed in Sec. III A, quantum-chemical molecular-scale calculations of the size of the dipole moments and their size and orientation distribution are nowadays well feasible, and various types of experimental studies can be used to experimentally determine the net preferential orientation (order parameter $S_{1}$ ). $S_{1}$ can, e.g., be conveniently deduced using UPS measurements from the layer-thickness dependence of the work function of a thin organic layer (see Sec. III A). The stray fields around the dipoles give rise to additional energetic disorder, with a certain spatial correlation. Well beyond the intermolecular distance, the dipolar stray fields are properly described using the relative dielectric permittivity of the organic materials, which is often close to 3 so that realistic uncertainties do not introduce a large error. We therefore expect that long-range effects of dipoles, e.g., the consequences of a net preferential orientation, are well described. The point-dipole approximation that is made in this work is expected to be less accurate at very short distances, on nearest-neighbor sites. The smeared-out nature of the charge density on the molecules and the spatially nonuniform polarizability of the local environment can give rise to a modification of the dipole-induced disorder and its spatial correlation, affecting the charge transport $[8,61]$. It will be of interest to study the possible consequences of including such higher-order effects on the device performance in 3D KMC simulations.

Future applications of this work are expected to include simulations of the performance of phosphorescent and thermally activated delayed fluorescent (TADF) OLEDs that are based on emitters with large static dipole moments. Some Ir-cored phosphorescent emitters have dipole moments that are well above $10 \mathrm{D}$ (see, e.g., Ref. [62]). Even when used at a concentration of only $10-20 \mathrm{~mol} \%$, as a guest in an emissive layer, the effect of the resulting energetic disorder on the device performance is expected to be significant.

\section{ACKNOWLEDGMENTS}

This work is supported by the National Key Research and Development Program of China (Grant No. 2017YFB 0404404), the leading talents of Guangdong Province Program (Grant No. 00201504), and the Guangdong Innovative Research Team Program (Grant No. 2013C102).

\section{APPENDIX. 3D KMC SIMULATIONS OF SENSE-LAYER EXPERIMENTS PROBING THE EMISSION PROFILE}

In this Appendix, we use 3D KMC simulations to investigate to what extent sense-layer experiments such as those that have been carried out in Ref. [34] on the device that is studied in this paper may be expected to provide accurate information about the shape of the emission profile. The experiments in Ref. [34] use the red sensitizer bis[2-(3,5-dimethylphenyl)-4-methylquinoline](tetrametylheptanedionate)iridium(III), abbreviated as $\operatorname{Ir}(\mathrm{mphmq})_{2}(\mathrm{tmd})$, with an emission wavelength of $604 \mathrm{~nm}$ (photon energy $2.05 \mathrm{eV}$ ) and a photoluminescence efficiency in PMMA of $87 \%$ [63]). In the experiments, a 0.5 -nm layer of $5 \mathrm{wt} \%$ of $\operatorname{Ir}(\mathrm{mphmq})_{2}(\mathrm{tmd})$ was codeposited in the EML, at distances of $0,5,10$, and $15 \mathrm{~nm}$ from the interface with the pure CBP hole transport layer. In a CBP matrix, this corresponds to a concentration of $2.9 \mathrm{~mol} \%$. Exciton transfer from $\operatorname{Ir}(\mathrm{ppy})_{2}$ (acac) to this red emitter leads to a distinct red component of the emission spectrum with an intensity that is expected to depend on the local green emission from the OLED. In order to minimize a possible effect of the sense layer on the charge transport, the concentration of red emitters should be taken very small. It was verified in Ref. [34] that the effect of the sense layers on the current density was indeed very small (of the order of the experimental sample-to-sample variation of at most a factor 1.5-2). The Förster radius for the transfer of triplet excitons from $\operatorname{Ir}(\mathrm{ppy})_{2}(\mathrm{acac})$ to 
$\operatorname{Ir}(\mathrm{mphmq})_{2}$ (tmd) was from a spectral overlap study found to be $2.5 \mathrm{~nm}$. One may expect that the maximum attainable spatial resolution is determined in part by this Förster radius.

We mimic such a sense-layer experiment by carrying out 3D KMC simulations for a device without TPBi dipoles, using the set of material parameters that is given in Sec. III B. In the simulations, we replace 1-nm layers of the two-component host-guest system, at various distances from the interface with the CBP hole transport layer, by a three-component host-guest-guest system [6.5 mol\% $\operatorname{Ir}\left(\right.$ ppy)2(acac) and $1.5 \mathrm{~mol} \% \operatorname{Ir}(\mathrm{mphmq})_{2}$ (tmd) in CBP]. This results in a layer structure that is as essentially equivalent to that in Ref. [34]. The radiative decay rate of the sense molecules is assumed to be $6.67 \times 10^{5} \mathrm{~s}^{-1}$, close to the experimental value of $6.59 \times 10^{5} \mathrm{~s}^{-1}$ [63]. Nonradiative decay is neglected. The Förster radius for triplet transfer from $\operatorname{Ir}(\mathrm{ppy})_{2}$ (acac) to $\operatorname{Ir}(\mathrm{mphmq})_{2}$ (tmd) is taken equal to the experimental value of $2.5 \mathrm{~nm}$. All other excitonic interaction parameters involving $\operatorname{Ir}(\mathrm{mphmq})_{2}(\mathrm{tmd})$ are taken equal to those involving $\operatorname{Ir}(\mathrm{ppy})_{2}(\mathrm{acac})$.

The energy-level scheme for the device without sense layer is given in Fig. 2 (dashed curves). The HOMO and LUMO energy of $\operatorname{Ir}(\mathrm{mphmq})_{2}(\mathrm{tmd})$ are not well known. A HOMO energy of $-4.7 \mathrm{eV}$ is obtained in Ref. [64] from cyclic voltammetry $(\mathrm{CV})$ experiments (oxidation potential measurement). The LUMO energy is in that work taken equal to $-2.9 \mathrm{eV}$. However, it is known that the ionization potential of molecules in a solution, as obtained from $\mathrm{CV}$ experiments, can differ from the thin-film value [65]. In order to study the sensitivity of the sensed emission profile to the frontier orbital energy of the sense molecules, we carry out simulations for four sets of HOMO and LUMO energies $(A)-(D)$. In set $A$, the HOMO energy is taken equal to the value of $-4.7 \mathrm{eV}$ that was obtained in Ref. [64]. The LUMO energy is taken equal to $-1.9 \mathrm{eV}$, which is obtained by adding an estimated triplet exciton binding energy of $1.0 \mathrm{eV}$ to the LUMO energy value of $-2.9 \mathrm{eV}$ that was given in Ref. [64]. For this set of HOMO and LUMO energies, strong hole trapping can happen on the sense molecules, whereas no electron trapping is expected. In sets $B-D$, the HOMO energy is downshifted in equal steps of $0.3 \mathrm{eV}$ to $-5.6 \mathrm{eV}(D)$. The LUMO energy is obtained from the HOMO energy by adding the triplet energy $(2.05 \mathrm{eV})$ and a triplet binding energy of $1.05 \mathrm{eV}$, similar to the value of $1.0 \mathrm{eV}$ that we assume for $\operatorname{Ir}(\mathrm{ppy})_{2}(\mathrm{acac})$. Table III gives an overview of all parameters.

Figures 8(a) and 8(b) show the calculated current density as a function of the sense-layer position, obtained from simulations at 7 and $11 \mathrm{~V}$, respectively. The dashed line reference current density, obtained without a sense layer. The width of the gray-colored zone indicates its statistical uncertainty. Both figures reveal for set $A$ a decrease of the current density of $10 \%-20 \%$ when the sense layer
TABLE III. Overview of material parameters characterizing $\operatorname{Ir}(\mathrm{mphmq})_{2}(\mathrm{tmd})$, used in the sense-layer simulations.

\begin{tabular}{llc}
\hline \hline Parameter & Description & Value \\
\hline$E_{T}$ & Triplet exciton energy & $2.05 \mathrm{eV}$ \\
$\Gamma_{r}$ & Radiative decay rate & $6.67 \times 10^{5} \mathrm{~s}^{-1}$ \\
$R_{F}$ & Förster radius $^{\mathrm{a}}$ & $2.5 \mathrm{~nm}$ \\
$E_{\mathrm{HOMO}}, E_{\mathrm{LUMO}}$ & Frontier orbital energies & \\
- Set $A$ & & $-4.7,-1.9 \mathrm{eV}$ \\
- Set $B$ & & $-5.0,-1.9 \mathrm{eV}$ \\
- Set $C$ & & $-5.3,-2.2 \mathrm{eV}$ \\
- Set $D$ & & $-5.6,-2.5 \mathrm{eV}$ \\
\hline \hline
\end{tabular}

${ }^{\text {a}}$ Förster radius for triplet transfer from $\operatorname{Ir}(\mathrm{ppy})_{2}$ (acac) to $\operatorname{Ir}(\mathrm{mphmq})_{2}(\mathrm{tmd})$. All other excitonic interaction parameters involving $\operatorname{Ir}(\mathrm{mphmq})_{2}(\mathrm{tmd})$ are taken equal to those involving $\operatorname{Ir}(\text { ppy })_{2}$ (acac) (see Table I).

is located relatively close to the anode side of the EML, where holes are still under way to the region of strong recombination zone close to the cathode side of the EML. From sets $A$ to $D$, the role of hole trapping becomes gradually less important. For sense layers that are located very close to the cathode side of the EML the current density is not significantly affected.

Figures 8(c) and 8(d) show the calculated normalized emission from the sense layers, as a function of their position in the EML, at 7 and $11 \mathrm{~V}$. The dashed curve gives the normalized emission profile. The figures show that for set $D$, for which the presence of the sense molecules least affects the current density, the emission profile that is deduced from the sense-layer experiments agrees most accurately with the actual emission profile. Only near the cathode interface of the EML the emission is underestimated. We attribute that, at least in part, to the nearby presence of the TPBi layer, from which no exciton transfer to the sense molecules occurs. The thickness of this interface zone, about $2 \mathrm{~nm}$, is similar to the Föster radius of 2.5 $\mathrm{nm}$ for exciton transfer to the sense molecules. For sets $A$ to $C$, for which hole trapping by the sense layers is found to give rise to a reduced current density, the emission profile that is deduced from sense-layer experiments would be expected to be more strongly distorted. The profile is less strongly peaked towards the cathode side of the EML.

The simulations thus show that already weak trapping of holes by the sense molecules, leading to a decrease of the current density of $10 \%-20 \%$ that is difficult to detect experimentally due to realistic sample-to-sample variations, can lead to a significant distortion of the shape of the sensed emission profile. Even though the experiments are still very valuable as a means to detect a nonuniformity of the emission profile, the sensed profile can in such a case only be used qualitatively. 3D KMC simulations such as demonstrated here can be used to obtain a more accurate interpretation of the sense-layer results. 

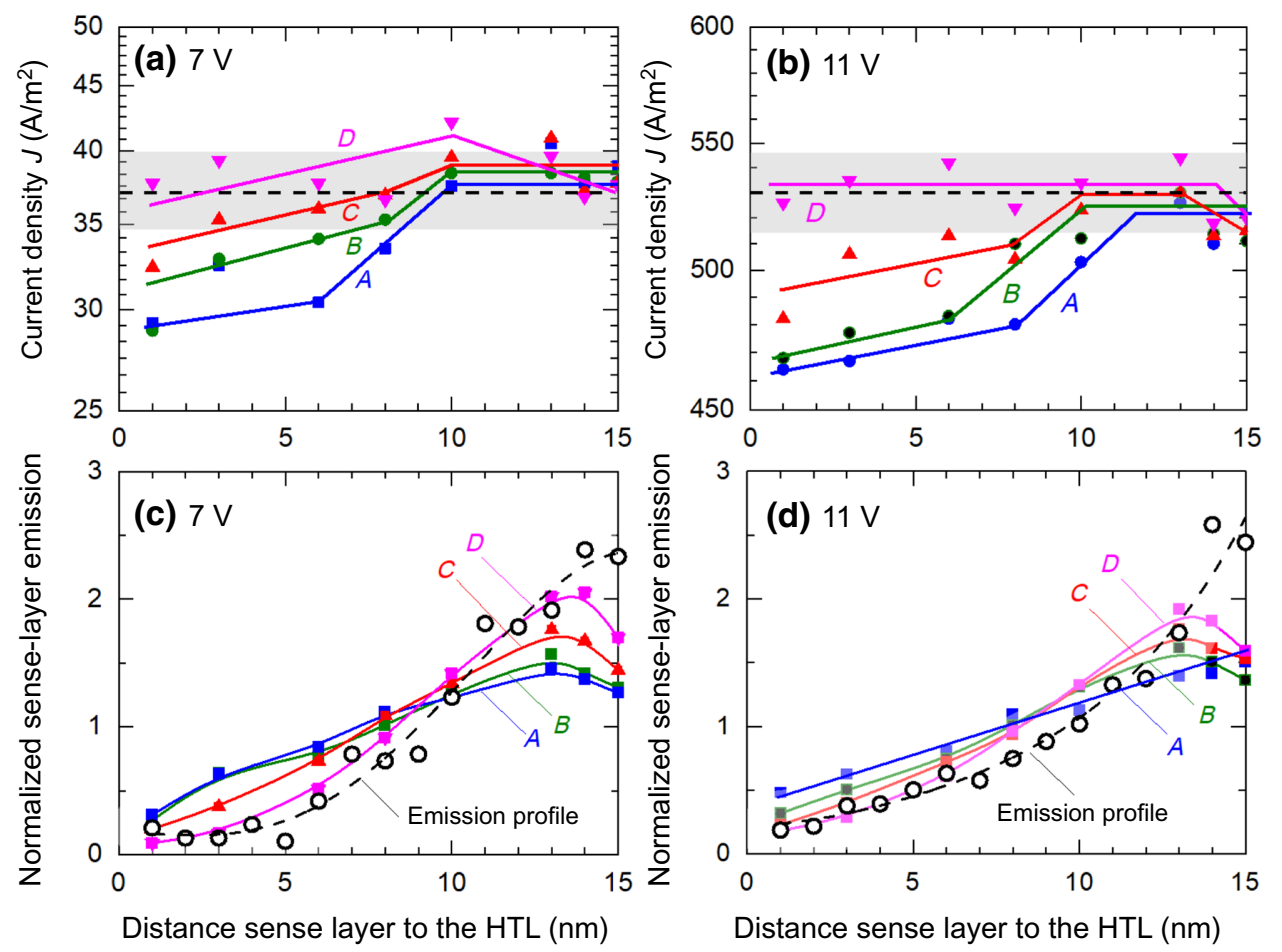

FIG. 8. Results of 3D KMC simulations of sense-layer studies of the emission profile of the device that is shown in Fig. 2, for four sets of frontier orbital energies ( $A-D$, see Table II) of the sense molecules. Dipole moments are not included; the simulations are carried out using the set of parameters that is discussed in Sec. V A. (a),(b) Effect on the current density at $7 \mathrm{~V} \mathrm{(a)}$ and $11 \mathrm{~V}$ (b) of adding a small concentration of sense molecules at various positions in the EML. The dashed line gives the result without the sense molecules, with an uncertainty that is given by the width of the gray zones. (c),(d) The (red) emission from the sense molecules at $7 \mathrm{~V}$ (c) and $11 \mathrm{~V}$ (e) as a function of their position in the EML. The dashed curves give the (green) $\operatorname{Ir}(\mathrm{ppy})_{2}(\mathrm{acac})$ emission in the absence of the sense molecules, obtained from 3D KMC simulations. The emission intensities are normalized such that their average value is equal to unity.

For the system that is studied in this work, the results that are shown in Fig. 8 indicate that the actual nonuniformity of the emission profile is stronger than as would be concluded from the sense-layer experiments when the HOMO energy level of $\operatorname{Ir}(\mathrm{mphmq})_{2}(\mathrm{tmd})$ is equal to the value that has been given in Ref. [34] (set $A$ of our simulations). That can explain, qualitatively, the difference in shape between the profiles that have been deduced from sense-layer experiments [Fig. 4(c)] and from our 3D KMC simulations [Fig. 4(e)]. Giving a more quantitative analysis would require obtaining more certainty about the frontier orbital energies of $\operatorname{Ir}(\mathrm{mphmq})_{2}(\mathrm{tmd})$ and carrying out simulations that include the TPBi dipole moments.

[1] P. M. Borsenberger and H. Bässler, Concerning the role of dipolar disorder on charge transport in molecularly doped polymers, J. Chem. Phys. 95, 5327 (1991).

[2] A. Dieckmann, H. Bässler, and P. M. Borsenberger, An assessment of the role of dipoles on the density of states function of disordered molecular solids, J. Chem. Phys. 99, 8136 (1993).
[3] R. H. Young, Dipolar lattice model of disorder in random media analytical evaluation of the Gaussian disorder model, Phil. Mag. B 72, 435 (1995).

[4] G. G. Malliaras, Y. Shen, D. H. Dunlap, H. Murata, and Z. H. Kafafi, Nondispersive electron transport in $\mathrm{Alq}_{3}$, Appl. Phys. Lett. 79, 2582 (2001).

[5] S. Novikov and A. Vannikov, Cluster structure in the distribution of the electrostatic potential in a lattice of randomly oriented dipoles, J. Phys. Chem. 99, 14573 (1995).

[6] D. H. Dunlap, P. E. Parris, and V. M. Kenkre, ChargeDipole Model for the Universal Field Dependence of Mobilities in Molecularly Doped Polymers, Phys. Rev. Lett. 77, 542 (1996).

[7] M. Bouhassoune, S. van Mensfoort, P. Bobbert, and R. Coehoorn, Carrier-density and field-dependent chargecarrier mobility in organic semiconductors with correlated Gaussian disorder, Org. Electron 10, 437 (2009).

[8] P. Kordt and D. Andrienko, Modeling of spatially correlated energetic disorder in organic semiconductors, J. Chem. Theory Comput. 12, 36 (2016).

[9] S. Berleb, W. Brütting, and G. Paasch, Interfacial charges and electric field distribution in organic hetero-layer lightemitting devices, Org. Electron 1, 41 (2000).

[10] E. Ito, Y. Washizu, N. Hayashi, H. Ishii, N. Matsuie, K. Tsuboi, Y. Ouchi, Y. Harima, K. Yamashita, and K. Seki, 
Spontaneous buildup of giant surface potential by vacuum deposition of $\mathrm{Alq}_{3}$ and its removal by visible light irradiation, J. Appl. Phys. 92, 7306 (2002).

[11] Y. Noguchi, Y. Miyazaki, Y. Tanaka, N. Sato, Y. Nakayama, T. D. Schmidt, W. Brütting, and H. Ishii, Charge accumulation at organic semiconductor interfaces due to a permanent dipole moment and its orientational order in bilayer devices, J. Appl. Phys. 111, 114508 (2012).

[12] P. Friederich, V. Rodin, F. von Wrochem, and W. Wenzel, Built-in potentials induced by molecular order in amorphous organic thin films, ACS Appl. Mater. Interfaces 10, 1881 (2018).

[13] A. J. Hofmann, S. Züfle, K. Shimizu, M. Schmid, V. Wessels, L. Jäger, S. Altazin, K. Ikegami, M. R. Khan, D. Neher, H. Ishii, B. Ruhstaller, and W. Brütting, Dipolar Doping of Organic Semiconductors to Enhance Carrier Injection, Phys. Rev. Appl. 12, 064052 (2019).

[14] Y. Noguchi, W. Brütting, and H. Ishii, Spontaneous orientation polarization in organic light-emitting diodes, Jpn. J. Appl. Phys. 58, SF0801 (2019).

[15] Y. Noguchi, K. Osada, K. Ninomiya, H. D. Gunawardana, K. R. Koswattage, and H. Ishii, Influence of intermolecular interactions on the formation of spontaneous orientation polarization in organic semiconducting films, J. Soc. Inform. Disp. 29, 29 (2021).

[16] N. H. Yamanaka and T. C. Adachi, Slow recombination of spontaneously dissociated organic fluorophore excitons, Nat. Commun. 10, 5748 (2010).

[17] J. S. Bangsund, J. R. Van Sambeek, N. M. Concannon, and R. J. Holmes, Sub-turn-on exciton quenching due to molecular orientation and polarization in organic light-emitting devices, Sci. Adv. 6, eabb2659 (2020).

[18] S. Reineke, G. Schwartz, K. Walzer, M. Falke, and K. Leo, Highly phosphorescent organic mixed films: The effect of aggregation on triplet-triplet annihilation, Appl. Phys. Lett. 94, 163305 (2009).

[19] C. Murawski, C. Elschner, S. Lenk, S. Reineke, and M. C. Gather, Investigating the molecular orientation of $\operatorname{Ir}(\mathrm{ppy})_{3}$ and $\operatorname{Ir}(\mathrm{ppy})_{2}$ (acac) emitter complexes by X-ray diffraction, Org. Electron 53, 198 (2018).

[20] M. Mesta, M. Carvelli, R. J. de Vries, H. van Eersel, J. J. M. van der Holst, M. Schober, M. Furno, B. Lüssem, K. Leo, P. Loebl, R. Coehoorn, and P. A. Bobbert, Molecularscale simulation of electroluminescence in a multilayer white organic light-emitting diode, Nat. Mater. 12, 652 (2013).

[21] M. Mesta, H. van Eersel, R. Coehoorn, and P. Bobbert, Kinetic Monte Carlo modeling of the efficiency roll-off in a multilayer white organic light-emitting device, Appl. Phys. Lett. 108, 133301 (2016).

[22] H. van Eersel, P. Bobbert, R. Janssen, and R. Coehoorn, Monte Carlo study of efficiency roll-off of phosphorescent organic light-emitting diodes: Evidence for dominant role of triplet-polaron quenching, Appl. Phys. Lett. 105, 143303 (2014).

[23] P. Kordt, J. J. M. van der Holst, M. A. Helwi, W. Kowalsky, F. May, A. Badinski, C. Lennartz, and D. Andrienko, Modeling of organic light emitting diodes: From molecular to device properties, Adv. Funct. Mater. 25, 1955 (2015).

[24] H. van Eersel, P. Bobbert, R. Janssen, and R. Coehoorn, Effect of Förster-mediated triplet-polaron quenching and triplet-triplet annihilation on the efficiency roll-off of organic light-emitting diodes, J. Appl. Phys. 119, 163102 (2016).

[25] Y. Shen and N. C. Giebink, Monte Carlo Simulations of Nanoscale Electrical Inhomogeneity in Organic Light-Emitting Diodes and its Impact on Their Efficiency and Lifetime, Phys. Rev. Appl. 4, 054017 (2015).

[26] S. Gottardi, M. Barbry, R. Coehoorn, H. van Eersel, and Efficiency loss processes in hyperfluorescent OLEDs, A kinetic Monte Carlo study, Appl. Phys. Lett. 114, 073301 (2019).

[27] J. Van der Holst, F. Van Oost, R. Coehoorn, and P. Bobbert, Electron-hole recombination in disordered organic semiconductors: Validity of the Langevin formula, Phys. Rev. B 80, 235202 (2009).

[28] Z. Gao, C. S. Lee, I. Bello, S. T. Lee, R.-M. Chen, T.-Y. Luh, J. Shi, and C. W. Tang, Bright-blue electroluminescence from a silyl-substituted ter-(phenylene-vinylene) derivative, Appl. Phys. Lett. 74, 865 (1999).

[29] C. Adachi, M. A. Baldo, S. R. Forrest, S. Lamansky, M. E. Thompson, and R. C. Kwong, High-efficiency red electrophosphorescence devices, Appl. Phys. Lett. 78, 1622 (2001).

[30] J. Meyer, S. Hamwi, T. Bülow, H.-H. Johannes, T. Riedl, and W. Kowalsky, Highly efficient simplified organic light emitting diodes, Appl. Phys. Lett. 91, 113506 (2007).

[31] S. Reineke, F. Lindner, G. Schwartz, N. Seidler, K. Walzer, B. Lüssem, and K. Leo, White organic light-emitting diodes with fluorescent tube efficiency, Nature 459, 234 (2009).

[32] P. O. Anikeeva, J. E. Halpert, M. G. Bawendi, and V. Bulović, Quantum dot light-emitting devices with electroluminescence tunable over the entire visible spectrum, Nano Lett. 9, 2532 (2009).

[33] Z. B. Wang, M. G. Helander, J. Qiu, D. P. Puzzo, M. T. Greiner, Z. W. Liu, and Z. H. Lu, Highly simplified phosphorescent organic light emitting diode with $>20 \%$ external quantum efficiency at $>10,000 \mathrm{~cd} / \mathrm{m} 2$, Appl. Phys. Lett. 98, 073310 (2011).

[34] B. Sim, C.-K. Moon, K.-H. Kim, and J.-J. Kim, Quantitative analysis of the efficiency of OLEDs, ACS Appl. Mater. Interf. 8, 33010 (2016).

[35] Z. B. Wang, M. G. Helander, J. Qiu, D. P. Puzzo, M. T. Greiner, Z. M. Hudson, S. Wang, Z. W. Liu, and Z. H. Lu, Unlocking the full potential of organic light-emitting diodes on flexible plastic, Nat. Photon 5, 753 (2011).

[36] P. Friederich, R. Coehoorn, and W. Wenzel, Molecular origin of the anisotropic dye orientation in emissive layers of organic light emitting diodes, Chem. Mater. 29, 9528 (2017).

[37] The 3D KMC tool Bumblebee is provided by Simbeyond B.V. (simbeyond.com).

[38] H. van Eersel, P. Bobbert, and R. Coehoorn, Kinetic Monte Carlo study of triplet-triplet annihilation in organic phosphorescent emitters, J. Appl. Phys. 117, 115502 (2015).

[39] J. Pumplin, Application of Sommerfeld-Watson transformation to an electrostatics problem, Am. J. Phys. 37, 737 (1969). 
[40] X. de Vries and R. Coehoorn, Vibrational mode contribution to the dielectric permittivity of disordered smallmolecule organic semiconductors, Phys. Rev. Mater. 4, 085602 (2020).

[41] M. Pabst, D. Sundholm, and A. Köhn, Ab initio studies of triplet-state properties for organic semiconductor molecules, J. Phys. Chem. C 116, 15203 (2012).

[42] M. Kröger, S. Hamwi, J. Meyer, T. Dobbertin, T. Riedl, W. Kowalsky, and H.-H. Johannes, Temperature-independent field-induced charge separation at doped organic/organic interfaces: Experimental modeling of electrical properties, Phys. Rev. B 75, 235321 (2007).

[43] N. Matsuura, H. Ishii, and Y. Tanaka, Demonstration of an electret generator using self-assembled electret for energy harvesting without any charging process, J. Phys.: Conf. Ser. 1407, 012116 (2019).

[44] Y. Tanaka, N. Matsuura, and H. Ishii, in 19th International Conference on Micro and Nanotechnology for Power Generation and Energy Conversion Applications (PowerMEMS) (2019), Vol. 49317, p. 1.

[45] Y. Tanaka, N. Matsuura, and H. Ishii, Self-assembled electret for vibration-based power generator, Sci. Rep. 10, 6648 (2020).

[46] G. Tirimbò, X. de Vries, C. H. L. Weijtens, P. A. Bobbert, T. Neumann, R. Coehoorn, and B. Baumeier, Quantitative predictions of photoelectron spectra in amorphous molecular solids from multiscale quasiparticle embedding, Phys. Rev. B 101, 035402 (2020).

[47] X. Crispin, V. Geskin, A. Crispin, J. Cornil, R. Lazzaroni, W. R. Salaneck, and J.-L. Brédas, Characterization of the interface dipole at organic/metal interfaces, J. Am. Chem. Soc. 124, 8131 (2002).

[48] G. Witte, S. Lukas, P. S. Bagus, and C. Wöll, Vacuum level alignment at organic/metal junctions: "cushion" effect and the interface dipole, Appl. Phys. Lett. 87, 263502 (2005).

[49] M. A. Baldo and S. R. Forrest, Interface-limited injection in amorphous organic semiconductors, Phys. Rev. B 64, 085201 (2001).

[50] M. Knupfer, Exciton binding energies in organic semiconductors, Appl. Phys. A 77, 623 (2003).

[51] H. Yoshida and K. Yoshizaki, Electron affinities of organic materials used for organic light-emitting diodes: A lowenergy inverse photoemission study, Org. Electron 20, 24 (2015).

[52] N. B. Kotadiya, A. Mondal, S. Xiong, P. W. M. Blom, D. Andrienko, and G.-J. A. H. Wetzelaer, Rigorous characterization and predictive modeling of hole transport in amorphous organic semiconductors, Adv. Electr. Mater. 4, 1800366 (2018).

[53] W. F. Pasveer, J. Cottaar, C. Tanase, R. Coehoorn, P. A. Bobbert, P. W. M. Blom, D. M. de Leeuw, and M. A. J. Michels, Unified Description of Charge-Carrier Mobilities in Disordered Semiconducting Polymers, Phys. Rev. Lett. 94, 206601 (2005).
[54] N. B. Kotadiya, A. Mondal, P. W. M. Blom, D. Andrienko, and G.-J. A. H. Wetzelaer, A window to trap-free charge transport in organic semiconducting thin films, Nat. Mater. 18, 1182 (2019).

[55] X. de Vries, P. Friederich, W. Wenzel, R. Coehoorn, and P. A. Bobbert, Triplet exciton diffusion in metalorganic phosphorescent host-guest systems from first principles, Phys. Rev. B 99, 205201 (2019).

[56] J. J. M. van der Holst, F. W. A. van Oost, R. Coehoorn, and P. A. Bobbert, Monte Carlo study of charge transport in organic sandwich-type single-carrier devices: Effects of coulomb interactions, Phys. Rev. B 83, 085206 (2011).

[57] W.-Y. Hung, T.-H. Ke, Y.-T. Lin, C.-C. Wu, T.-H. Hung, T.C. Chao, K.-T. Wong, and C.-I. Wu, Employing ambipolar oligofluorene as the charge-generation layer in time-offlight mobility measurements of organic thin films, Appl. Phys. Lett. 88, 064102 (2006).

[58] T. Tsuboi, S.-W. Liu, M.-F. Wu, and C.-T. Chen, Spectroscopic and electrical characteristics of highly efficient tetraphenylsilane-carbazole organic compound as host material for blue organic light emitting diodes, Org. Electron 10, 1372 (2009).

[59] T. van Woudenbergh, P. W. M. Blom, M. C. J. M. Vissenberg, and J. N. Huiberts, Temperature dependence of the charge injection in poly-dialkoxy-p-phenylene vinylene, Appl. Phys. Lett. 79, 1697 (2001).

[60] T. Isoshima, Y. Okabayashi, E. Ito, M. Hara, W. W. Chin, and J. W. Han, Negative giant surface potential of vacuum-evaporated tris(7-propyl-8-hydroxyquinolinolato) aluminum(iii) [Al(7-Prq) 3 ] film, Org. Electron 14, 1988 (2013).

[61] F. May, B. Baumeier, C. Lennartz, and D. Andrienko, Can Lattice Models Predict the Density of States of Amorphous Organic Semiconductors? Phys. Rev. Lett. 109, 136401 (2012).

[62] J. Lee, H.-F. Chen, T. Batagoda, C. Coburn, P. I. Djurovich, M. E. Thompson, and S. R. Forrest, Deep blue phosphorescent organic light-emitting diodes with very high brightness and efficiency, Nat. Mater. 15, 92 (2016).

[63] D. H. Kim, N. S. Cho, H.-Y. Oh, J. H. Yang, W. S. Jeon, J. S. Park, M. C. Suh, and J. H. Kwon, Highly efficient red phosphorescent dopants in organic light-emitting devices, Adv. Mater. 23, 2721 (2011).

[64] J. G. Fan, M. J. Kim, Q. P. B. Nguyen, R. Braveenth, H. M. Ko, J.-H. Han, G. W. Kim, J. H. Kwon, and K. Y. Chai, Synthesis and characterization of carbazole corebased small molecular-hole-transporting materials for red phosphorescent OLEDs, Bull. Korean Chem. Soc. 37, 1710 (2016).

[65] B. W. D’Andrade, S. Datta, S. R. Forrest, P. Djurovich, E. Polikarpov, and M. E. Thompson, Relationship between the ionization and oxidation potentials of molecular organic semiconductors, Org. Electron 6, 11 (2005). 